Research Article

\title{
Pseudodynamic Testing of Buildings Retrofitted with External Steel Reinforced Concrete Frames to Increase Lateral Strength for Earthquake Damage Prevention
}

\author{
Ju-Seong Jung ${ }^{1}$ and Kang-Seok Lee $\mathbb{1}^{2}$ \\ ${ }^{1}$ Department of Architectural Engineering, Hanyang University, Seoul, Republic of Korea \\ ${ }^{2}$ Department of Architectural Engineering, Hanyang University, Ansan, Republic of Korea \\ Correspondence should be addressed to Kang-Seok Lee; ksleenist@hanyang.ac.kr
}

Received 1 October 2019; Revised 14 May 2020; Accepted 12 June 2020; Published 16 July 2020

Academic Editor: Ivo Caliò

Copyright $\odot 2020$ Ju-Seong Jung and Kang-Seok Lee. This is an open access article distributed under the Creative Commons Attribution License, which permits unrestricted use, distribution, and reproduction in any medium, provided the original work is properly cited.

In this study, a new technique for seismic retrofitting via the attachment of an "external steel reinforced concrete frame" (ESRCF) system was developed to strengthen medium-to-low-rise reinforced concrete (RC) buildings. Two methods (bolting and welding) were developed to connect existing RC frames and external strengthening elements; these methods are technically and practically suited to various construction conditions. The retrofitting method developed in this study can be used to perform seismic strengthening construction, while residents continue to live within the building. The method is categorized as a "strength design approach" implemented via retrofitting, allowing the lateral ultimate load capacity of RC buildings, whose failure mode is shear, to be increased easily. Test specimens were designed based on an existing RC building in Korea lacking seismic data and then strengthened using the ESRCF system. Pseudodynamic and cyclic loading tests were conducted to verify the effects of seismic retrofitting. In total, four RC frame specimens were prepared: one nonstrengthened control specimen for the cyclic loading test, one nonstrengthened control specimen, one specimen strengthened with a welded ESRCF system, and one specimen strengthened with a bolted ESRCF system for the pseudodynamic test. The earthquake response behavior with use of the proposed method, in terms of the maximum response strength, response displacement, and degree of earthquake damage, is compared with a control RC frame. The test results indicated that both the bolting and welding methods used for connecting the existing RC frame to the ESRCF effectively increased the lateral ultimate strength, resulting in reduced response displacement of building structures under large-scale earthquake conditions.

\section{Introduction}

Earthquakes are among the most hazardous natural disasters and have frequently wrought devastation, both economically and on human life. Although the repeatability and macroscopic distribution of historic earthquakes can be predicted to a certain extent, it is impossible to predict precisely when and where an earthquake will occur. The destruction of building structures vividly demonstrates the severity of earthquake damage, as observed during a recent series of earthquakes. Long-term earthquake observation and research has improved our understanding of their behaviors, including patterns of damage to structures, and significant efforts have been made to mitigate earthquake damage to new buildings through seismic design. In particular, correlations between the degree of damage caused by earthquakes and the seismic resistance mechanisms of structures have been subjected to vigorous theoretical and experimental investigations. Procedures for calculating shear forces and dynamic responses based on the results of these studies have been adopted in seismic design codes and standards during recent decades, including the International Building Code [1], Building code requirements for structural concrete of the American Concrete Institute [2], Standard for structural calculation of reinforced concrete structures of the Architectural Institute of Japan [3], and the Korean Building Code [4].

Progress in seismic design has resulted in new buildings with improved prospects for satisfactory behavior during earthquakes. Innovations in seismic design methodologies have led designers to question the adequacy of the seismic 
behavior of existing buildings lacking seismic data, including those affected by recent destructive earthquakes such as the 2008 Sichuan earthquake in China, the 2010 Chile earthquake, the 2011 Van earthquake in Turkey, the 2011 Christchurch earthquake in New Zealand, the 2012 Great East Japan earthquake, the 2016 Central Italy earthquake, the 2016 Kumamoto earthquake in Japan, and the 2017 Pohang earthquake in South Korea. According to the results of previous investigations of earthquake damage, including those listed above, a large proportion of earthquake damage occurs in medium-to-low-rise buildings with fewer than six stories, especially reinforced concrete (RC) buildings lacking seismic data. Therefore, it is necessary to retrofit existing medium-to-low-rise RC buildings lacking seismic data to be resistant against earthquakes, and important existing buildings are required to be fully operational even under severe earthquake conditions.

Over the past three decades, rehabilitation procedures for existing RC buildings have been promoted, leading to the development of several seismic strengthening techniques to improve their seismic performance [5-9]. These methods include techniques to improve ultimate strength or deformation capability, as well as technology for vibration control. Most medium-to-low-rise RC buildings lacking seismic data feature column hoop spacing of approximately $30 \mathrm{~cm}$ or wider, resulting in a high risk of shear failure [5, 10-14]; [6]. Retrofitting methods that adopt independent construction techniques for improving ductility have been shown to be inefficient due to extremely inadequate ultimate horizontal strength. Retrofitting techniques that increase strength to improve the seismic performance of medium-to-low-rise RC buildings lacking seismic data are more efficient during shear. Such methods (e.g., use of external joints and frames) can be applied, while residents continue to live within the building, and are thus more economical [7, 15-17].

However, most conventional seismic retrofitting techniques used to increase the ultimate strength of RC buildings, such as adding new shear walls to existing frames, steel braces to existing frames, side walls (wing walls) to existing columns, and steel panels to existing frames, and involve internal connections and postinstalled anchors. Seismic strengthening based on internal connections requires expansion into additional areas, which limits building space during construction. With such strengthening work, the retrofitting period can increase indefinitely due to the difficulty of securing adequate work space. In addition, precise construction is required for postinstalled anchor systems. Thus, to overcome the drawbacks of the conventional seismic retrofitting technology used to increase ultimate strength, there is an urgent need for a new external internal connection technique that will allow residents to live within the building during construction.

The main purpose of this study was to develop a new method based on external connections for seismic strengthening of existing medium-to-row rise RC frame systems lacking seismic data. The proposed technique uses an "external steel reinforced concrete frame" (ESRCF) system. The seismic performance of the new ESRCF strengthening system was investigated, with the goal of overcoming the drawbacks associated with conventional seismic retrofitting technology, to increase the ultimate strength of existing medium-to-row rise RC frame systems lacking seismic data. Bolting and welding methods for connecting existing RC frames to external strengthening elements were also tested in this study. These connection techniques are technically and practically suitable for various construction conditions. The retrofitting method developed in this study allows for seismic retrofitting construction, while residents continue to live within the building. The proposed method represents a strength design approach implemented via retrofitting to easily increase the ultimate lateral load capacity of RC buildings, which have shear failure mode.

On the other hand, the optimal method for evaluating the seismic performance of a test structure, e.g., a framing system subjected to earthquake ground motions, is a fullscale structural test performed on a shaking table. Generally, such a test is not possible within current experimental facilities and will never be practical for most large structures. Several alternative approaches have been developed, including shaking table tests of reduced-scale structural models. The drawbacks of such reduced-scale tests are obvious; many structures cannot be adequately represented by reduced-scale structural models.

The pseudodynamic test method was developed to conduct realistic experimental tests on full-scale structures subjected to earthquake ground motions [18]. This test uses an online computer and associated test instrumentation to monitor and control a structure, such that displacement thereof closely resembles the consequences of real seismic excitation. The pseudodynamic test is as realistic as shaking table-based testing, where discretization of the model is feasible; its advantages over shaking tables include (a) versatility, where it allows for detailed observation of the specimen during the test, (b) the ability to test full- and large-scale models, thus circumventing the problem of dynamic similitude, (c) lack of requirement for physical structures, because the method uses a numerical model, (d) greatly reduced equipment, installation, and operation costs, (e) circumventing the problems associated with the interactions of a shaking table with heavy specimens, and (f) improved actuator control and data acquisition reliability due to the relatively slow rate of loading. In principle, the test can be performed in real time; however, physical limitations of the instrumentation dictate that the test must be conducted step-by-step, i.e., pseudodynamically. Shaking table tests may be more appropriate in cases where strain rate effects are significant and/or in distributed parameter systems. Experimental measurements of restoring forces are performed during the test. These measured forces are then fed into the computer, together with a set of mathematical equations for inertial response characteristics, to determine the structural displacement that would occur as a consequence of a given ground acceleration. This procedure is superior to quasistatic testing because nonlinear structural characteristics are based on instantaneous experimental feedback rather than hypothetical mathematical models. Pseudodynamic testing differs from classical computerbased structural dynamic simulations in that the latter 
depend on experimentally measured restoring forces rather than on restoring forces computed using a mathematical model.

In this study, test specimens strengthened with the ESRCF system were designed and fabricated on the basis of typical existing RC buildings constructed prior to the seismic code revision in Korea. A pseudodynamic test, as previously stated, was conducted to evaluate the seismic performance of the proposed ESRCF system, and the effectiveness of bolting and welding methods for connecting existing RC frames with external strengthening systems. The test results were compared with those of a control RC frame in terms of maximum response strength, response displacement, and degree of earthquake damage, according to earthquake levels specified by the KBC [4]. Prior to the pseudodynamic test, a cyclic loading test was performed to estimate ultimate and yield shear load-carrying capacity, deformation, and hysteresis in the lateral load-drift relationship of the control RC frame. Hysteresis properties must be evaluated to determine test variables for pseudodynamic tests. In total, four RC frame specimens were prepared: one nonstrengthened control specimen for the cyclic loading test, one nonstrengthened control specimen, one specimen strengthened with a welded ESRCF system, and one specimen strengthened with a bolted ESRCF system for the pseudodynamic test.

\section{Overview of the ESRCF Strengthening System}

The widely used chemical lap-splicing method, which employs steel reinforcing bars (rebar) to connect existing RC frames to external strengthening elements, inevitably leads to cracks in the concrete surface during an earthquake (Figure 1) due to insufficient ductility capacity of the joint concrete. This cracking prevents transmission of the earthquake load from the existing structure to the strengthening system; thus, successful seismic retrofitting is unlikely [7]. The ESRCF system was proposed to improve structural integrity compared with the conventional chemical lap-splicing method.

This study also proposes bolting and welding methods for connecting existing RC frames to external strengthening elements. These methods are technically and practically suited to various construction conditions, including in terms of work space and workability.

2.1. The Welded ESRCF Strengthening System. Figure 2 depicts detailed diagram of a connection element and technical details of the welded ESRCF strengthening system. As shown in Figure 2, the welded ESRCF strengthening system is composed of (A) an existing structure, (B) an anchor plate to strengthen the existing structure, $(\mathrm{C})$ an anchor bolt for jointing, (D) a steel frame, (E) a connection plate, (F) a plate to strengthen the connection, and $(\mathrm{G})$ epoxy resin; the system is finished with concrete. The main features of the welded ESRCF strengthening system are the anchor plate (B), and the welding of the connection plate (E) to another plate to strengthen the connection (F). This welded connection integrates the existing structure with the reinforcing member. Table 1 lists the construction steps. The welded ESRCF system is superior in terms of workability because welding of connection elements can be conducted during construction work, but is disadvantageous in terms of constructability and work space.

\subsection{The Bolted ESRCF Strengthening System. Figure 3 shows} detailed diagram of a connection element and technical details of the bolted ESRCF strengthening system. As shown in Figure 3, the bolted ESRCF system consists of (A) an existing structure, (B) a steel plate to strengthen the existing structure, (C) an anchor for fixing the steel plate, (D) a steel frame, (E) a connecting device, (F) a bolt for fixing the slit plate, and $(\mathrm{G})$ epoxy resin; the system is finished with concrete. Important features of the bolted ESRCF strengthening system include the bolting of the steel plate (B) to the connecting device, and (E) the fixing bolt of the slit plate $(F)$, which is installed along with the steel frame (D). This bolted connection results in integration of the existing structure with the reinforcing member. Table 2 lists the construction steps. The bolting method is superior in terms of constructability, but workability is reduced in terms of jointing, setting up, and other factors.

\subsection{Postconstruction Image of the Proposed ESRCF System.} Figure 4 shows a postconstruction image of the proposed ESRCF system. The retrofitting method developed in this study can be implemented for seismic retrofitting construction, while residents continue to live within the building. The method represents a "strength design approach" implemented via retrofitting, to easily increase the ultimate lateral load capacity of RC buildings lacking seismic data, which have shear failure mode.

\section{Experimental Tests and Procedure}

3.1. Specimen Design and Fabrication. The main objective of the cycling loading and pseudodynamic tests was to verify the seismic strengthening effectiveness of the welded and bolted ESRCF systems via connecting existing RC frames to external strengthening members. Specimens were designed and fabricated based on the frame of a typical three-story Korean RC building [17], designed in the 1980s and lacking seismic data. The specified compressive strength of the concrete used in this building was $21 \mathrm{MPa}$, and the story height was $3,300 \mathrm{~mm}$. The structure consisted of RC frames with spandrel walls in the longitudinal direction, and infilled brick walls in the transverse direction. One story with one span of the exterior frame in the longitudinal direction, including the columns, beams, and spandrel walls of the structure, is shown in Figures 4 and 5. This building type was selected for testing because the seismic capacity of in-filled brick walls in the transverse direction is higher than that in the longitudinal direction $[5,6]$. The ratio of the dimension reduction of the specimens was approximately $60 \%$ (Table 3 ) when the size of the laboratory facility, actuator equipment, and capacity of the overhead crane were considered. 


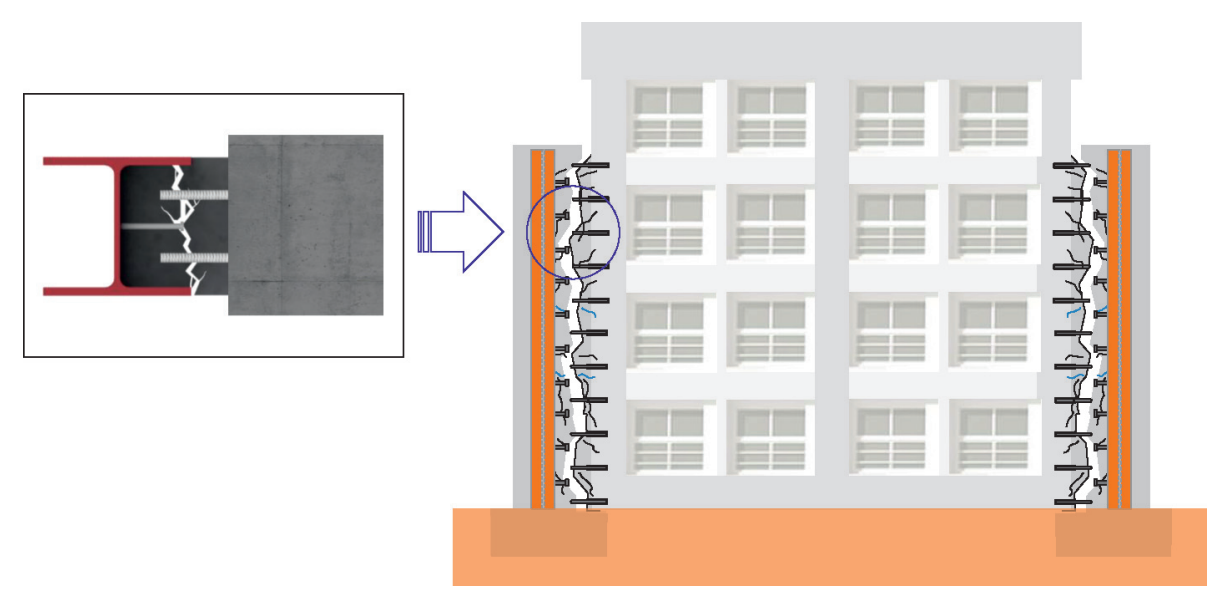

Figure 1: Drawbacks of the conventional chemical lap-splicing method (i.e., cracks in the concrete surface), which uses a steel rebar for connecting existing reinforced concrete (RC) frames to external strengthening elements.

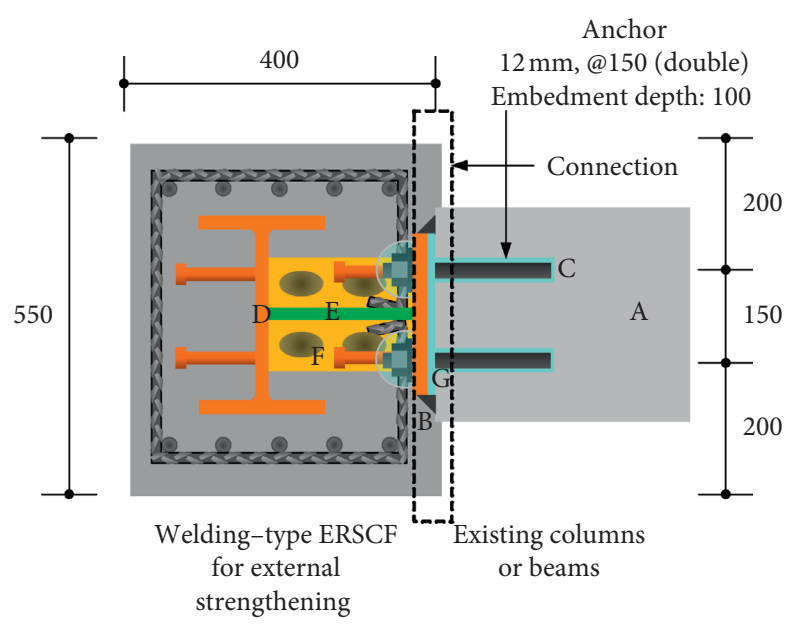

FIGURE 2: Detailed diagram of a connection element and technical details of the welded ESRCF strengthening system.

TABLE 1: Construction procedure for the welded ESRCF strengthening system.

Construction procedure
Boring of anchoring hall
Installation of anchor plate
Installation of connection device and frame (welding)
Fixing of anchor plate, epoxy sealing, injection, and filling
Installation of rebar, fixing concrete cast in place




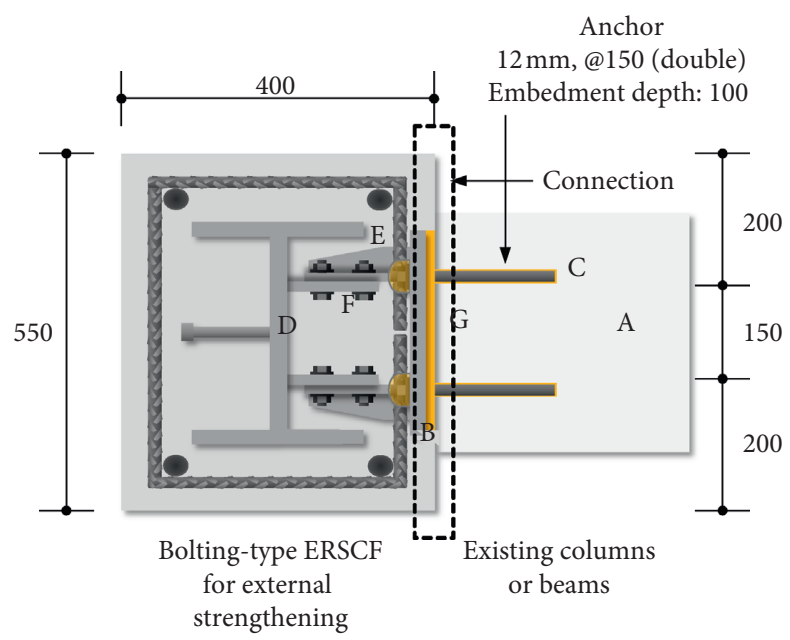

FIGURE 3: Detailed diagram of a connection element and technical details of the bolted ESRCF strengthening system.

TABLE 2: Construction procedure for the bolted ESRCF strengthening system.

Construction procedure
Boring of anchoring hall
Installation of anchor plate
Tixing of anchor plate, epoxy sealing, injection, and filling
Installation of rebar, fixing concrete cast in place

The configurations of the scaled-down specimens are shown in Figures 5 and 6 . The purpose of the tests was to verify the seismic strengthening effectiveness of the ESRCF system, in terms of maximum response strength, response displacement, degree of earthquake damage, and hysteresis in the lateral load-drift relationship, compared with the control specimen. Specimens consisting of columns and beams were designed according to the guidelines for loadcarrying capacity of the JBDPA [6].

Table 3 lists the specifications of each specimen tested. In total, four test specimens were prepared: a control test specimen (nonstrengthened, C-RC) for the cyclic loading test, a control test specimen (nonstrengthened, PD-RC), test specimen strengthened with a welded ESRCF system (PDWESRCF), and test specimen strengthened with a bolted
ESRCF system (PD-BESRCF) for the pseudodynamic test. All specimens had identical dimensions and rebar arrangements. The column cross section was $300 \times 300 \mathrm{~mm}$, and the ratio of column clear height to depth $\left(h_{0} / D\right)$ was 4.7. Each column had an 8-D16-type SD400 main rebar, reinforced with D10 steel shear reinforcement bars at 250-mm intervals. Beams were fabricated for installation as external strengthening members; stiff stubs were installed at the tops of all specimens to secure the columns (Figure 5). The spandrel wall was $480 \mathrm{~mm}$ high and constructed using B-type brick (190 mm long $\times 90 \mathrm{~mm}$ wide $\times 57 \mathrm{~mm}$ thick). The compressive strength of the brick was $8 \mathrm{MPa}$ [17].

The ground motion acceleration in the pseudodynamic test was the north-south (NS) ground motion recorded at El Centro (Table 3) during the 1940 Imperial Valley earthquake, 


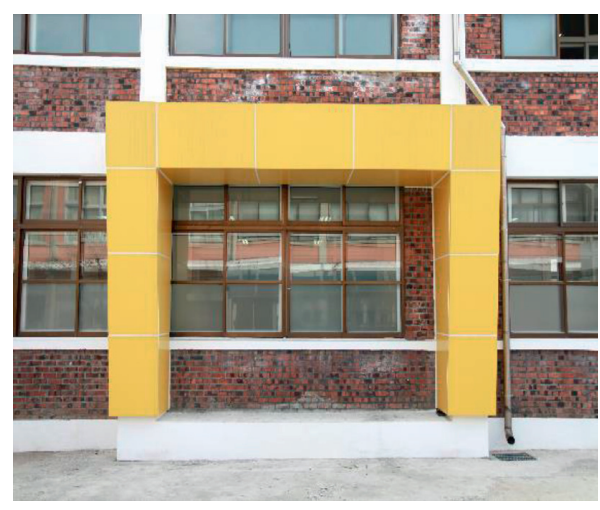

(a)

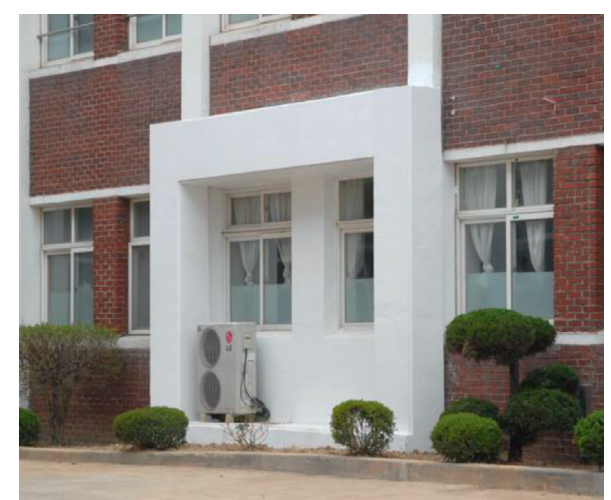

(b)

FIGURE 4: Postconstruction image of the external steel reinforced concrete frame (ESRCF) system.

TABLE 3: Summary of the test conditions for cyclic loading and pseudodynamic tests.

\begin{tabular}{lcccc}
\hline Specimen & Experimental method & Strengthening type & Clear span of columns $(\mathrm{mm})$ & Earthquake levels for pseudodynamic test (gal) \\
\hline C-RC & Cyclic loading & - & 1,400 & - \\
PD-RC & Pseudodynamic & - & 1,400 & El centro (NS): 250, 300 \\
PD- & Pseudodynamic & Welding & 1,400 & El centro (NS): $300,400,500$ \\
WESRCF & Polting & 1,400 & El centro (NS): $300,400,500$ \\
PD-BESRCF & Pseudodynamic & B &
\end{tabular}

C-RC: nonstrengthened control specimen for the cyclic loading test; PD-RC: nonstrengthened control specimen for the pseudodynamic test; PD-WESRCF: specimen strengthened using the welded ESRCF system for the pseudodynamic test; PD-BESRCF: specimen strengthened using the bolted ESRCF system for the pseudodynamic test; NS: north-south.

which has been widely used to verify seismic performance in various building systems. The magnitude of ground motion was normalized to acceleration values of $250,300,375$, and 450 gal. Figure 7 shows time history records of the normalized ground motion accelerations used in the pseudodynamic test, respectively, together with their acceleration response spectrum. The ground motion acceleration of 250 and $300 \mathrm{gal}$ approximately corresponds to the seismic load in Zone-1 with $S_{\mathrm{D}^{-}}$and $S_{\mathrm{E}}$ soil conditions, under which two-thirds of the earthquake ground motion have a $2 \%$ probability of exceedance within 50 years (i.e., a 2,500-year return period), as specified by the KBC [4]. Ground motions of 375 and $450 \mathrm{gal}$ were also used, to assess the seismic strengthening effectiveness of the ESRCF system in the context of a very strong earthquake, under which all earthquake ground motion has a $2 \%$ probability of exceedance within 50 years. Before the pseudodynamic test, a cyclic loading test was conducted to estimate the lateral load-drift relationship of an $\mathrm{RC}$ frame lacking seismic data (C-RC specimen).

3.2. Material Properties. As previously stated, the specified compressive strength of the concrete of the targeting building was $21 \mathrm{MPa}$. Cylindrical axial compression tests yielded a value of $21.5 \pm 1.2 \mathrm{MPa}$. The test results compare well to the design compressive strength of concrete.

The tensile strength of the steel rebar was $400 \mathrm{MPa}$. We used two rebar diameters: D10 for the shear reinforcement rebar and D16 for the main specimen rebar. The tensile strength of the steel rebar, measured using a universal testing machine (UTM), was $471.0 \pm 1.33 \mathrm{MPa}$ for D10 and $516.0 \pm 1.55 \mathrm{MPa}$ for D16.

\subsection{Joint Connection Design of the ESRCF Strengthening} System. The type, embedding depth, and anchor space of the joint connections for the ESRCF strengthening system were based on the anchor design provided by the JBDPA [6]. Table 4 lists the calculation results, and Figures 3 and 4 show the diameter, spacing, and embedding depth of the anchors for the welded and bolted ESRCF strengthening systems, respectively. Figure 5 shows the configuration of a test specimen strengthened using the ESRCF system, based on the joint connection results shown in Table 4.

Based on these results, the joint connection anchors had a diameter (D) of $12 \mathrm{~mm}$ and spacing of $150 \mathrm{~mm}$, in double arrangement. A total of 30 anchors were used in each column, with 12 anchors within each segment of $0.2 L_{\mathrm{C}}\left(L_{\mathrm{C}}\right.$ : column lengths) from the top and bottom of the column, and 18 anchors within each segment of $0.6 L_{C}$. A total of 38 anchors were used within the beam: 16 anchors within each segment of $0.2 L_{\mathrm{B}}$ (beam lengths) from the left and right of the beam, and 22 anchors within each segment of $0.6 L_{\mathrm{B}}$. The strength of the joint connections was approximately 4.0 -fold higher for the $0.2 L_{\mathrm{C}}$ and $0.2 L_{\mathrm{B}}$ segments, and 1.2-fold higher for the $0.6 L \mathrm{c}$ and $L_{\mathrm{B}}$ segments, versus the lateral resisting force on the ESRCF strengthening frame (Table 4). The ESRCF system proposed in this study was able to resist the seismic load when used with the existing RC frame.

\subsection{Description of the Pseudodynamic Testing System Used in} This Study. A simplified schematic diagram of the pseudodynamic testing system used in this study is shown in Figure 8, together with the test configuration. The system can 


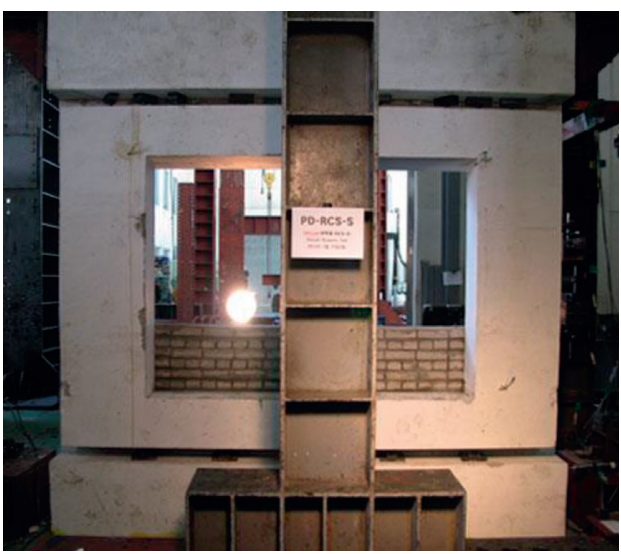

(a)

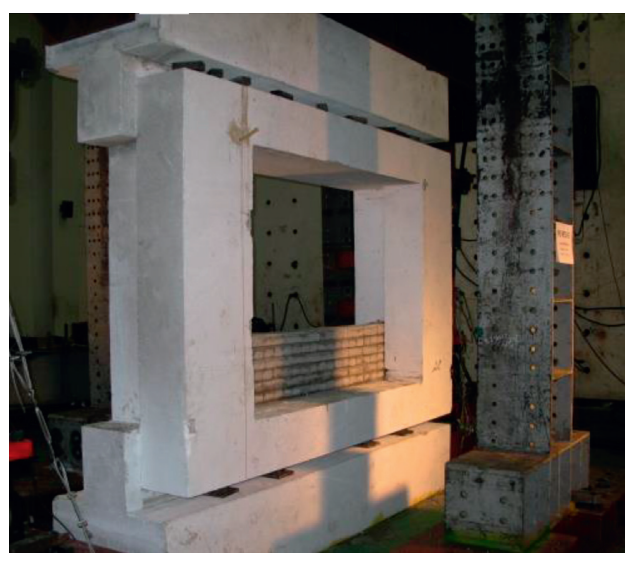

(c)

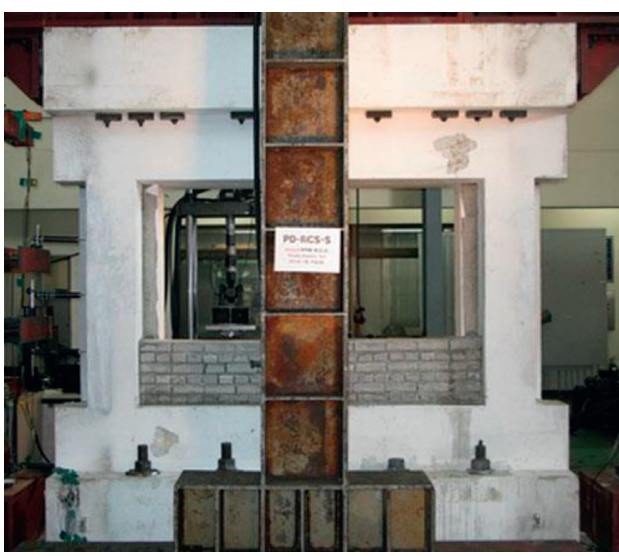

(b)

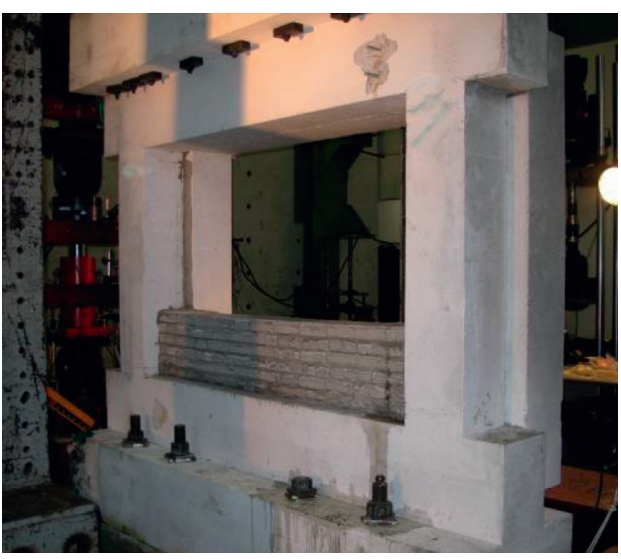

(d)

FIGURE 5: Detailed configuration of the test specimens strengthened with the ESRCF system. (a) Front view; (b) rear view; (c) front isometric view; and (d) rear isometric view.

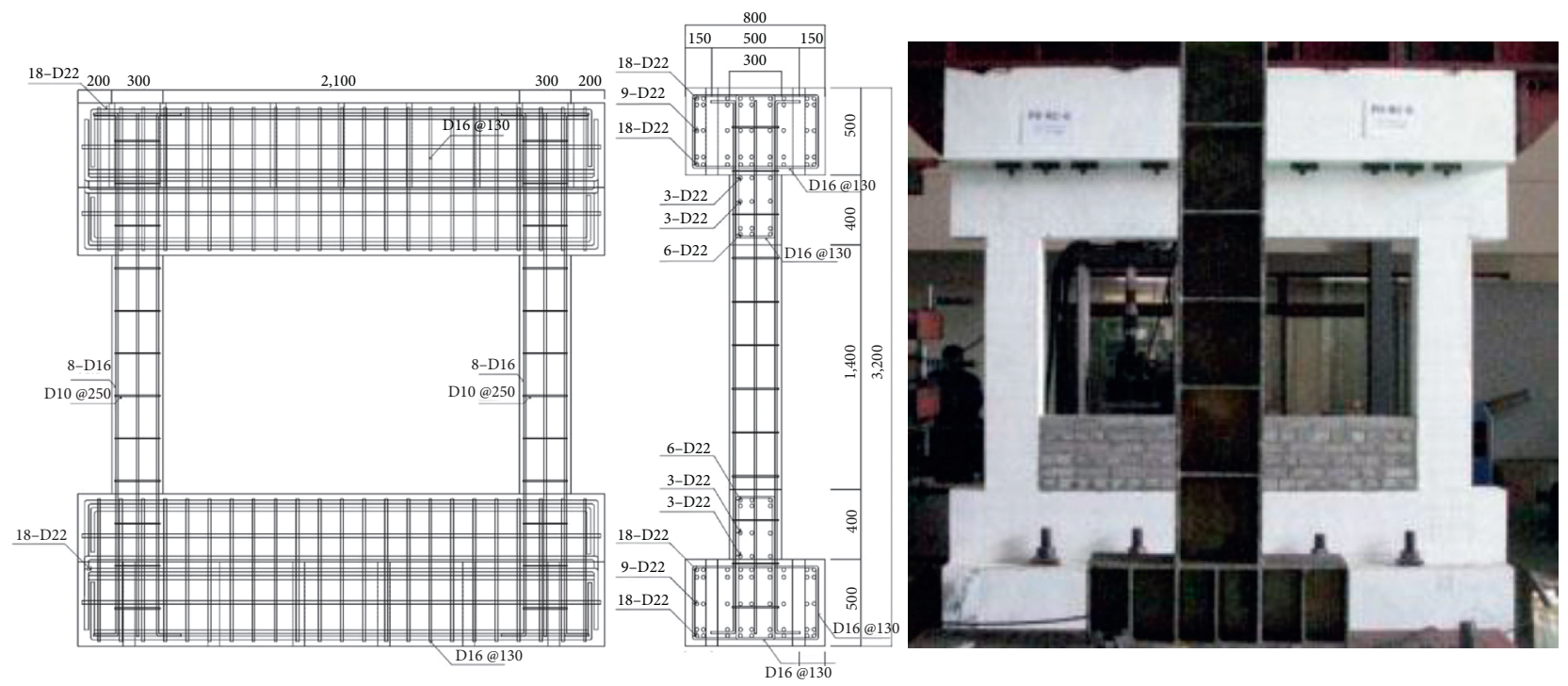

FiguRE 6: Detailed configuration of the control specimen. 


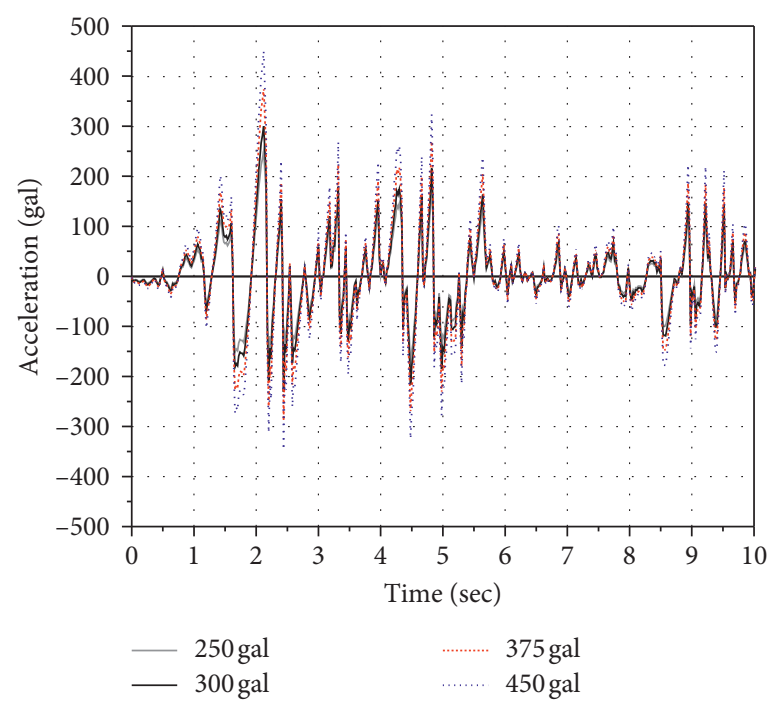

(a)

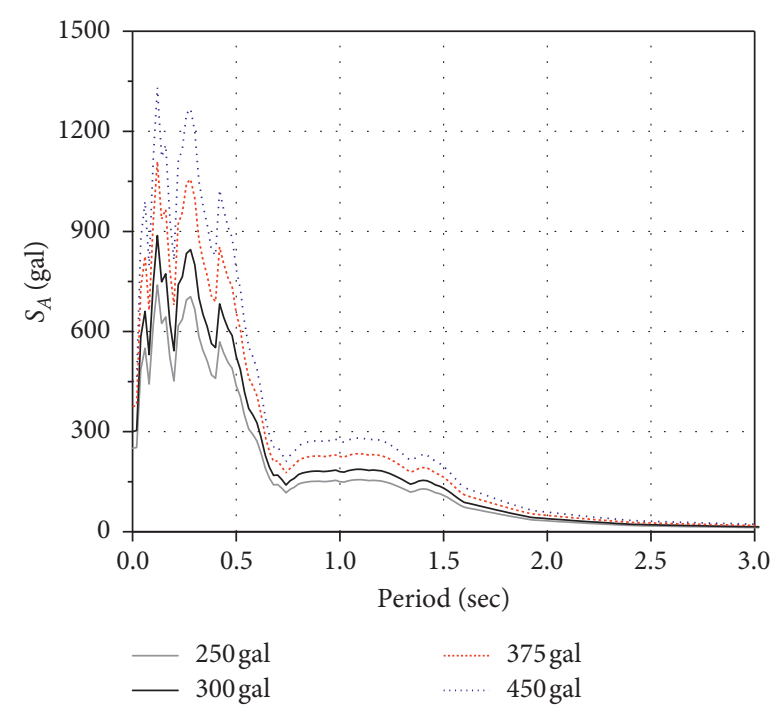

(b)

Figure 7: Ground motion accelerations used in the pseudodynamic test. (a) Time history records and (b) acceleration response spectrum.

TABLE 4: ESRCF system joint connection test results.

\begin{tabular}{|c|c|c|c|c|c|}
\hline Member & Member length & Number of anchors & $V_{f}(\mathrm{kN})$ & $\sum V_{a c}(\mathrm{kN})$ & Safety ratio \\
\hline \multirow{2}{*}{ Beam } & $0.2 L_{\mathrm{B}}$ & 8 & 23.7 & 107.5 & 4.5 \\
\hline & $0.6 L_{\mathrm{B}}$ & 22 & 256.2 & 311.5 & 1.2 \\
\hline \multirow{2}{*}{ Column } & $0.2 L_{\mathrm{C}}$ & 6 & 23.7 & 80.6 & 3.4 \\
\hline & $0.6 L_{\mathrm{C}}$ & 18 & 208.8 & 254.8 & 1.2 \\
\hline
\end{tabular}

$L_{\mathrm{B}}$ : beam length; $L_{\mathrm{C}}$ : column length; $V_{f}$ : lateral resisting force on the ESRCF strengthening frame; $\sum V_{a c}$ : strength of joint connections using anchors.

be expressed as a single degree of freedom (SDF) structure. During the test, the computed displacement response is imposed on the specimen via hydraulic actuators. The actual restoring forces are physically measured during the test and used by the control computer to calculate the displacement. Data transformation is achieved using an analog-to-digital/ digital-to-analog converter (DA-16A) [19]. Filtering and amplification are performed to ensure reliable results and accurate closed-loop control.

We used a pseudodynamic testing program [20] to calculate displacement based on the equation of motion of the SDF structure, as determined by the control computer:

$$
m \ddot{y}(t)+c \dot{y}(t)+r(t)=-m \ddot{u},
$$

where $y$ is the relative displacement of the mass with respect to its base, $c$ is the damping coefficient, $r$ is the hysteresis restoring force, and $\ddot{u}$ is the base acceleration.

The time integration scheme adopted for solving the equation of motion was an adaptive time-stepping algorithm developed by Shing et al. [21], based on the $\alpha$-method proposed by Hilber et al. [22]. The algorithm for numerical integration of the pseudodynamic test is expressed as follows:

$$
m a_{i+1}+(1+\alpha) c v_{i+1}-\alpha c v_{i}+(1+\alpha) r_{i+1}-\alpha r_{i}=(1+\alpha) f_{i+1}-\alpha f_{i}
$$

with

$$
\begin{gathered}
y_{i+1}=y_{i}+\Delta t v_{i}+\Delta t^{2}\left[\left(\frac{1}{2}-\beta\right) a_{i}+\beta a_{i+1}\right], \\
v_{i+1}=v_{i}+\Delta t\left[(1-\gamma) a_{i}+\gamma a_{i+1}\right],
\end{gathered}
$$

where, $y_{i}, v_{i}$, and $a_{i}$ are the mass displacement, velocity, and acceleration at time $i \Delta t$, respectively, $\Delta t$ is the integration time step, $r_{i}$ is the restoring force, and $f_{i}$ is the external force (i.e., $-m \ddot{u}_{i}$ ). For a linearly elastic structure, $r_{i}=k y_{i}$, in which $k$ is the elastic stiffness of the structure. $\alpha, \beta$, and $\gamma$ are parameters that govern the numerical properties of the algorithm, which is unconditionally stable when $-0.5 \leq \alpha \leq 0$, $\beta=(1-\alpha)^{2} / 4$, and $\gamma=(1 / 2)-\alpha$.

The displacement response for the next time step was calculated using equations (1)-(4), based on the stiffness $(k)$, mass $(\mathrm{m})$, and stiffness proportional damping coefficient $(c=2 \xi \sqrt{k \cdot m})$, in which the damping ratio $\xi$ was assumed to be 0.03 (i.e., $3 \%$ of the critical damping value). The initial stiffness (elastic stiffness), as for the input data for the pseudodynamic testing program, was derived from the results of the cyclic loading test, as will be described. A 2,000-kN hydraulic actuator was used to apply the lateral load. The horizontal displacement used to calculate the displacement response was measured using a 300-mm linear variable differential transformer (LVDT). Each column was subjected to a constant vertical load of $219 \mathrm{kN}$, which is half of the total weight of one 


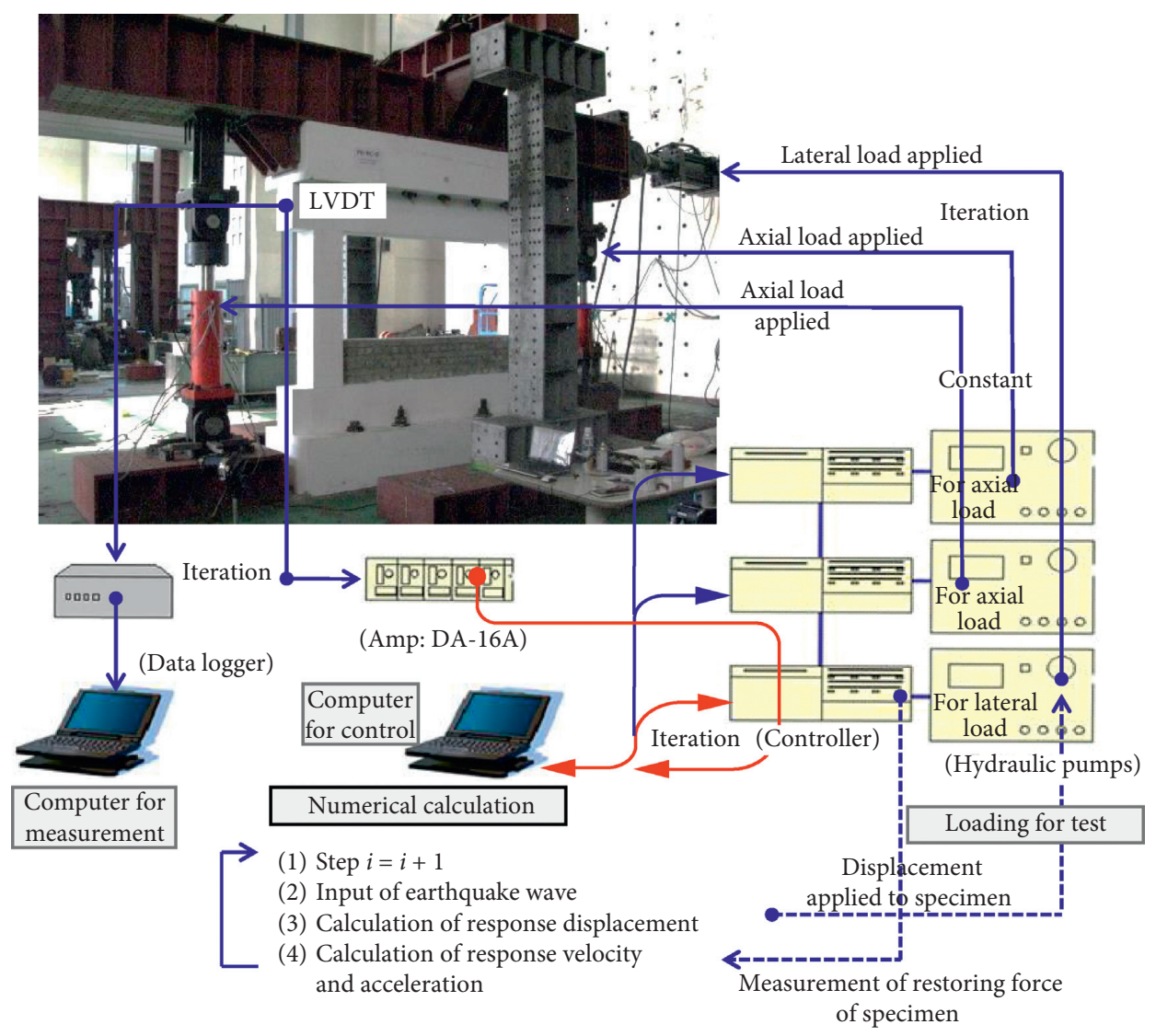

Figure 8: Simplified schematic diagram of the pseudodynamic testing system used in this study.

span of the exterior frame in the longitudinal direction, using two $1,000-\mathrm{kN}$ oil jacks.

The ground motion acceleration for the pseudodynamic test was the NS ground motion recorded at El Centro during the 1940 Imperial Valley earthquake. As stated previously, the magnitude of the ground motion was normalized to acceleration values of $250,300,375$, and 450 gal, based on the KBC [4]. Prior to the pseudodynamic test, a cyclic loading test was performed to estimate the ultimate and yield shear load-carrying capacity and deformation, as well as hysteresis in the lateral load-drift relationship of the C-RC specimen. A cyclic loading scheme with predetermined displacement was used. Load cycles were designed to have antisymmetric moments in the lateral direction and were repeated three times at lateral drift angles (R, \%) of $0.04 \%$ (loading step: $1,0.56 \mathrm{~mm}$ ), $0.1 \%$ (loading step: $2,1.4 \mathrm{~mm}$ ), $0.2 \%$ (loading step: $3,2.8 \mathrm{~mm}$ ), $0.25 \%$ (loading step: $4,3.5 \mathrm{~mm}$ ), $0.33 \%$ (loading step: $5,4.62 \mathrm{~mm}$ ), $0.4 \%$ (loading step: $6,5.6 \mathrm{~mm}$ ), $0.5 \%$ (loading step: $7,7 \mathrm{~mm}$ ), $0.67 \%$ (loading step: $8,9.38 \mathrm{~mm}$ ), $1 \%$ (loading step: $9,14 \mathrm{~mm}$ ), $1.5 \%$ (loading step: 10, $21 \mathrm{~mm}$ ), 2\% (loading step: 11, $28 \mathrm{~mm}$ ), $3.0 \%$ (loading step: 12, $42 \mathrm{~mm}$ ), and 5\% (loading step: 13, $70 \mathrm{~mm}$ ). Figure 9 shows the experimental configuration for the pseudodynamic and cyclic loading tests.

\section{Results and Discussion}

4.1. Load-Displacement Relationships and Estimation of Earthquake Damage. The ultimate load capacity $\left(V_{u}\right)$ of the nonstrengthened control C-RC specimen was a positive load of $311 \mathrm{kN}$, with lateral drift of $19.5 \mathrm{~mm}(R=1.4 \%)$, and a negative load of $-282 \mathrm{kN}$ was accompanied by lateral drift of $18.0 \mathrm{~mm}(R=1.3 \%)$. At the ultimate state, the failure mode was shear, which represents useful information for determining the failure mechanisms of existing Korean RC buildings lacking seismic data. Figure 10 shows load-displacement curves for the C-RC control specimen during the cyclic loading test. Table 5 lists its mechanical characteristics, i.e., shear strength and displacement, under positive and negative loads.

Figure 11 shows the lateral response load-displacement relationship of the $\mathrm{PD}-\mathrm{RC}$ control specimen during the pseudodynamic test under the 250 and 300 gal NS ground motion recorded at El Centro during the 1940 Imperial Valley earthquake; this approximately corresponds to the seismic load in Zone-1 under $S_{\mathrm{D}}$ and $S_{\mathrm{E}}$ soil conditions, under which two-thirds of the earthquake ground motion have a $2 \%$ probability of exceedance within 50 years $(2,500$ year return period), as specified in the $\mathrm{KBC}$ [4]. The figure shows an envelope curve describing the lateral load-displacement relationship of the C-RC control specimen following the cyclic loading test.

Figures 12-14 show the lateral response load-displacement relationships of the PD-WERCF and PD-BERCF specimens during pseudodynamic testing at 300, 375, and 450 gal, respectively. We also used ground motion of 375 and 450 gal to assess the seismic strengthening effectiveness of 


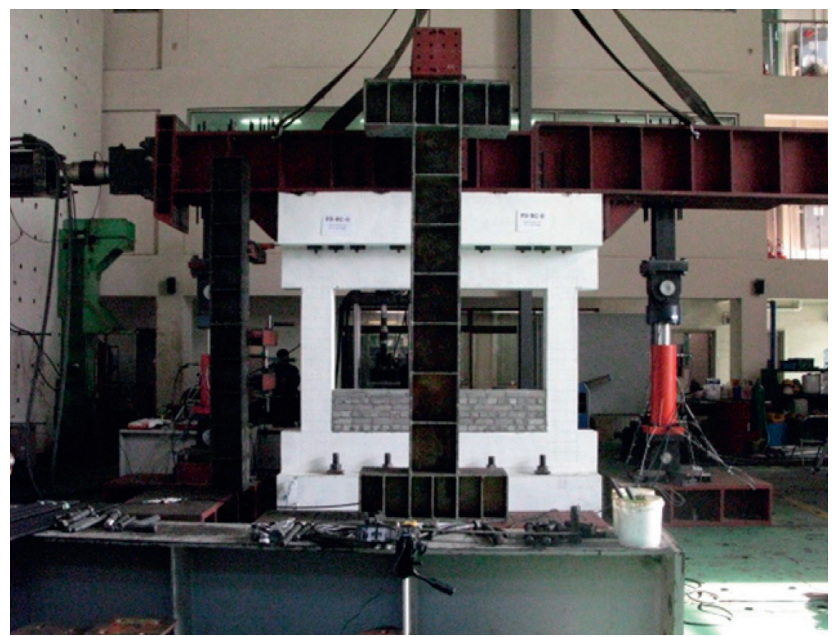

FIgURE 9: Test configuration.

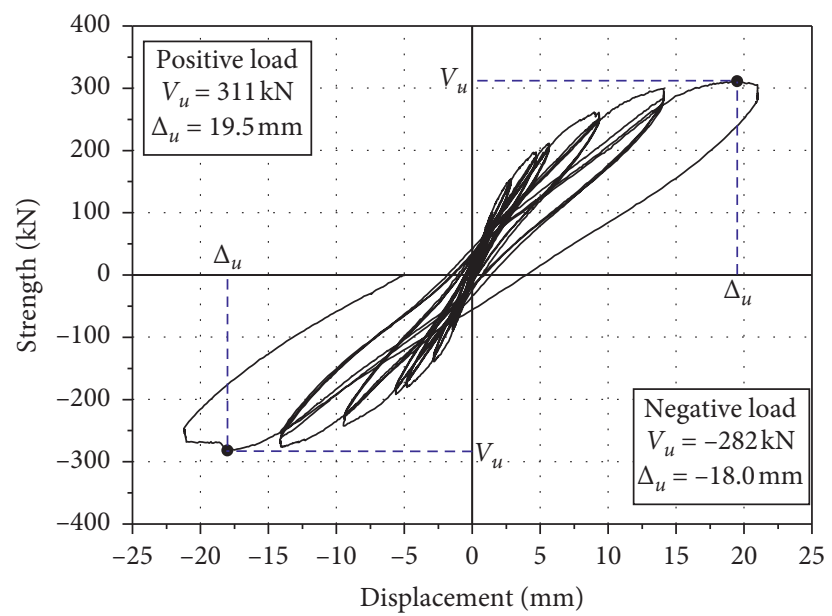

FiguRE 10: Lateral load-displacement curve for the C-RC specimen during the cyclic loading test. $V_{u}$ : ultimate shear strength $\Delta_{u}$ : displacement at the ultimate point.

TABLE 5: Mechanical characterization of the control C-RC specimen during the cyclic loading test.

\begin{tabular}{lcccc}
\hline Specimen & $\begin{array}{c}\text { Load } \\
\text { direction }\end{array}$ & $\begin{array}{c}\text { Ultimate shear strength } V_{u} \\
(\mathrm{kN})\end{array}$ & $\begin{array}{c}\text { Displacement at the ultimate point } \Delta_{u} \\
(\mathrm{~mm})\end{array}$ & $\begin{array}{c}\text { Failure mode at the ultimate } \\
\text { state }\end{array}$ \\
\hline \multirow{2}{*}{ C-RC } & Positive & 311 & 19.5 & Shear failure \\
& Negative & -282 & -18.0 & S \\
\hline
\end{tabular}

the ESRCF system under very strong earthquake conditions, where all earthquake ground motion has a $2 \%$ probability of exceedance within 50 years. The response load-displacement relationship of the PD-RC control specimen at $300 \mathrm{gal}$ is characterized by an envelope curve for the C-RC control specimen following the cyclic loading test. Table 6 presents a comparison of maximum response strength, maximum response displacement, and degree of earthquake damage in the pseudodynamic test for the PD-RC specimen at 250 and $300 \mathrm{gal}$, and for the PD-WERCF and PD-BERCF specimens at 300,375 , and 450 gal. The degree of earthquake damage was estimated based on the technique for postearthquake damage evaluation of RC buildings proposed by the JBDPA [23] and Maeda et al. [11].

The maximum earthquake response of the PD-RC control specimen for an earthquake ground motion of 250 gal was observed under a load of $305 \mathrm{kN}$ with lateral displacement of $11.9 \mathrm{~mm}$, which was classified as heavy earthquake damage according to the JBDPA [23]. For ground motion of $300 \mathrm{gal}$, the same control specimen showed a maximum response strength of $310 \mathrm{kN}$ with lateral displacement of $16.7 \mathrm{~mm}$, which is practically equivalent to drift; this is compared with the displacement at the ultimate point under positive load of $19.5 \mathrm{~mm}$ for the C-RC specimen 


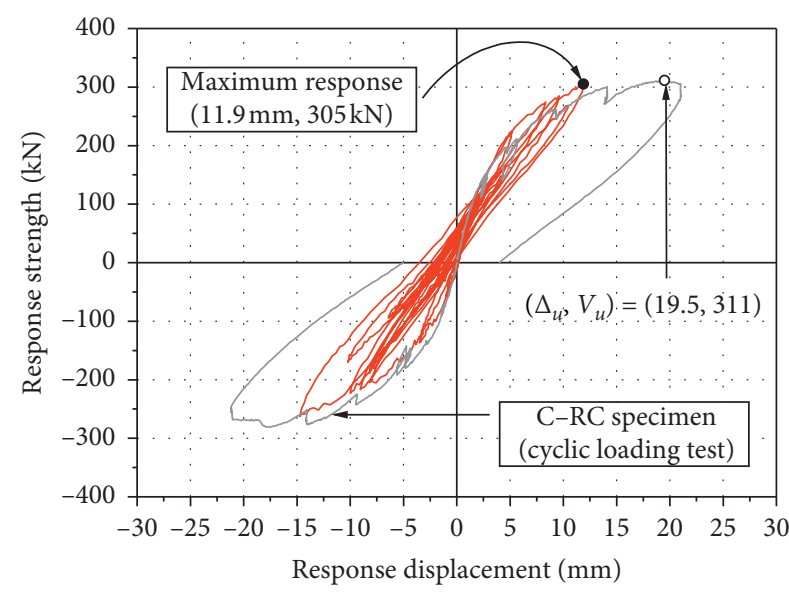

(a)

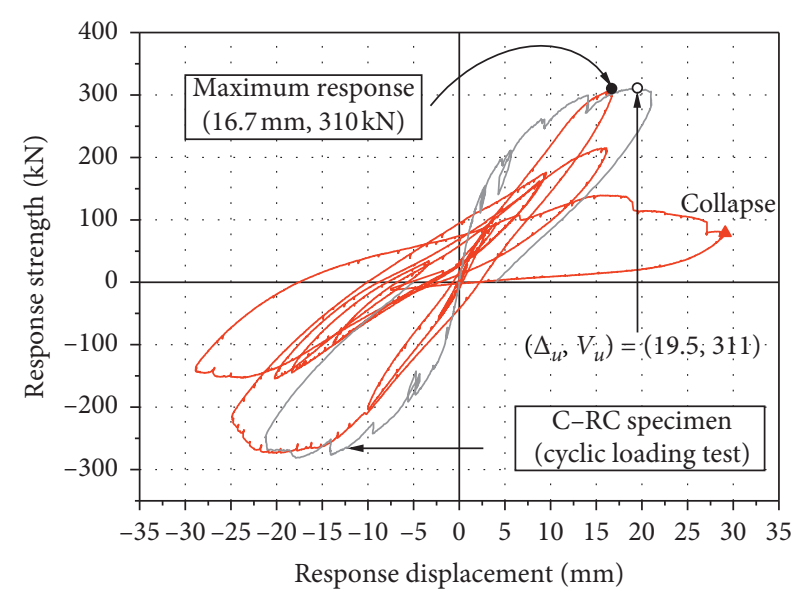

(b)

FIGURE 11: Lateral response load-displacement relationships for the control PD-RC specimen during pseudodynamic testing at (a) 250 gal and (b) 300 gal.

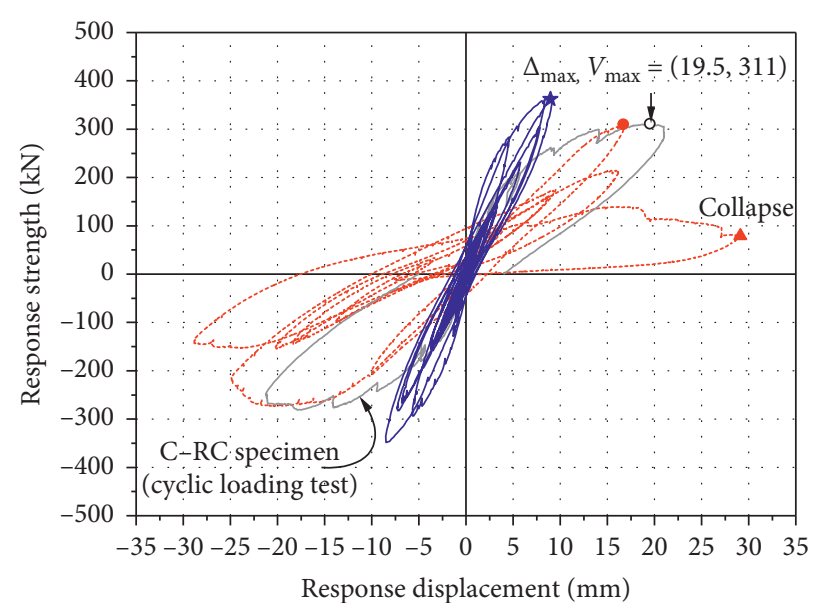

- Maximum response

PD-RC specimen (psedodynamic test, $300 \mathrm{gal}$ )

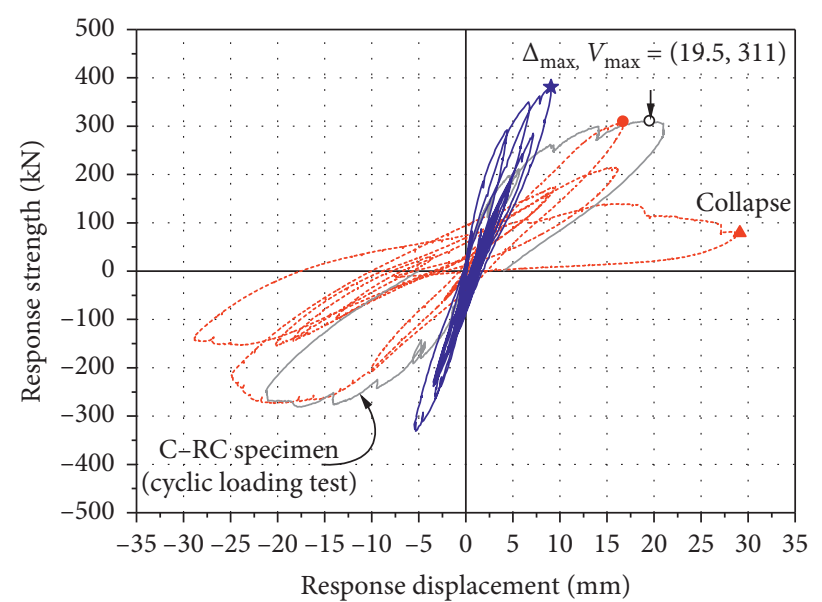

- Maximum response $(16.7 \mathrm{~mm}, 310 \mathrm{kN})$

...-.... PD-RC specimen (psedodynamic test, $300 \mathrm{gal}$ ) $\star \quad$ Maximum response

- PD-BERCF specimen (psedodynamic test, $300 \mathrm{gal}$ )

(a)

FIGURE 12: Lateral response load-displacement relationships for specimens strengthened with the ESRCF system during pseudodynamic testing at 300 gal. (a) PD-WERCF; (b) PD-BERCF.

during the cyclic loading test. The control specimen collapsed at the tops of both columns at $5.4 \mathrm{~s}$, following the application of a positive load of $80 \mathrm{kN}$, with lateral displacement of $28.9 \mathrm{~mm}$ during an earthquake ground motion of 300 gal.

For an earthquake ground motion of 300 gal, the PDWERCF specimen showed a maximum response strength of $362 \mathrm{kN}$ with displacement of $9.0 \mathrm{~mm}$, suffering slight damage in the form of minor flexural and shear cracks, whereas the PD-RC control specimen sustained shear collapse. The earthquake response behavior of, and degree of damage to, the PD-BERCF specimen strengthened with the bolted ESRCF system was similar to that of the PD-WERCF specimen. The PD-BERCF specimen showed a maximum response strength of $381 \mathrm{kN}$ with lateral displacement of $9.1 \mathrm{~mm}$ at $300 \mathrm{gal}$. Among the strengthened specimens, the strength ratio (Table 6) at the maximum response to $300 \mathrm{gal}$ was 1.2-fold higher on average than that of the control PDRC specimen.

Under severe earthquake ground motion ( 375 gal), the PD-WERCF specimen showed a maximum earthquake response at a load of $472 \mathrm{kN}$ with lateral displacement of $21.1 \mathrm{~mm}$; this was similar to that of the PD-BERCF specimen, with a load of $435 \mathrm{kN}$ and lateral displacement of $23.6 \mathrm{~mm}$. The strength ratio of the reinforced specimens at $375 \mathrm{gal}$ was 1.45 -fold higher than that of the control specimen. For both 


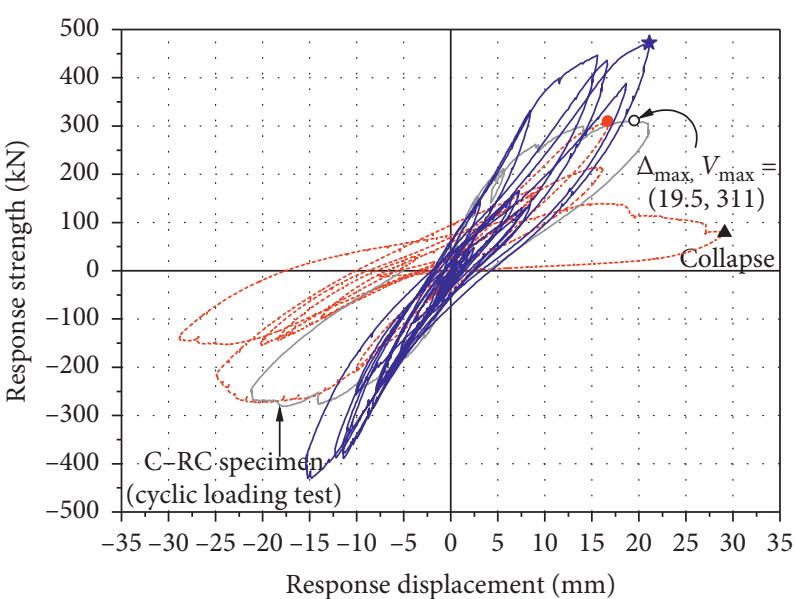

- Maximum response

-..-.-.. PD-RC specimen (psedodynamic test, $300 \mathrm{gal})$

(a)
PD-WERCF specimen (psedodynamic test 375 gal) a)

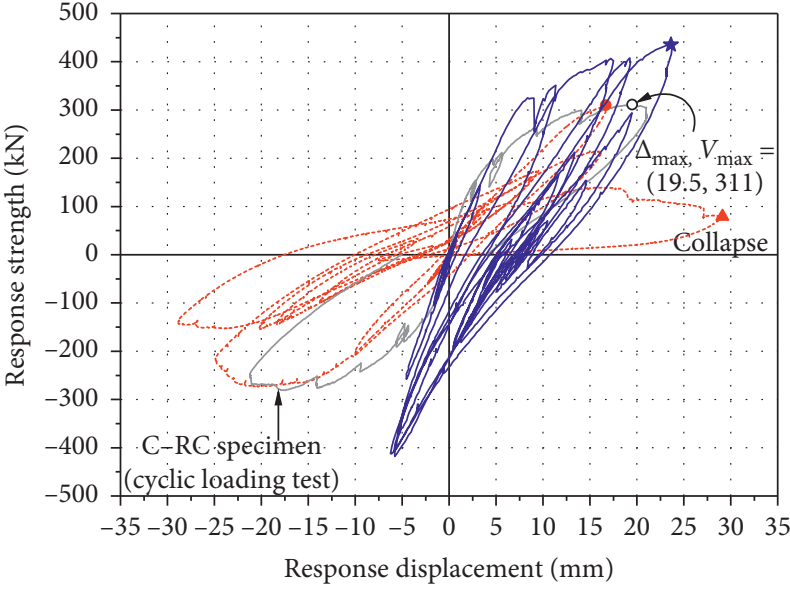

- Maximum response $(16.7 \mathrm{~mm}, 310 \mathrm{kN})$

$\star \quad$ Maximum response PD-RC specimen (psedodynamic test, 300 gal)

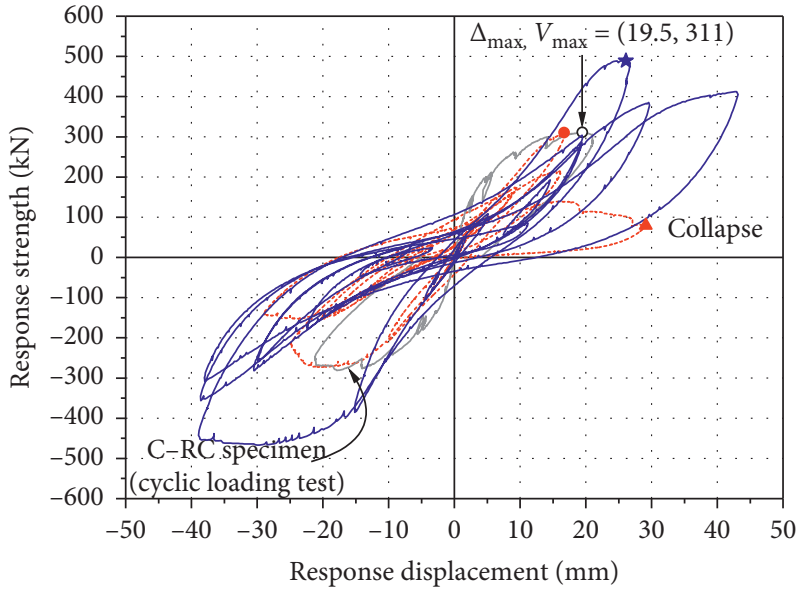

- Maximum response $(16.7 \mathrm{~mm}, 310 \mathrm{kN})$ PD-RC specimen (psedodynamic test, $300 \mathrm{gal})$

$\star \quad$ Maximum response (26.1 mm, 489kN)

- PD-WERCF specimen (psedodynamic test, $450 \mathrm{gal}$ )

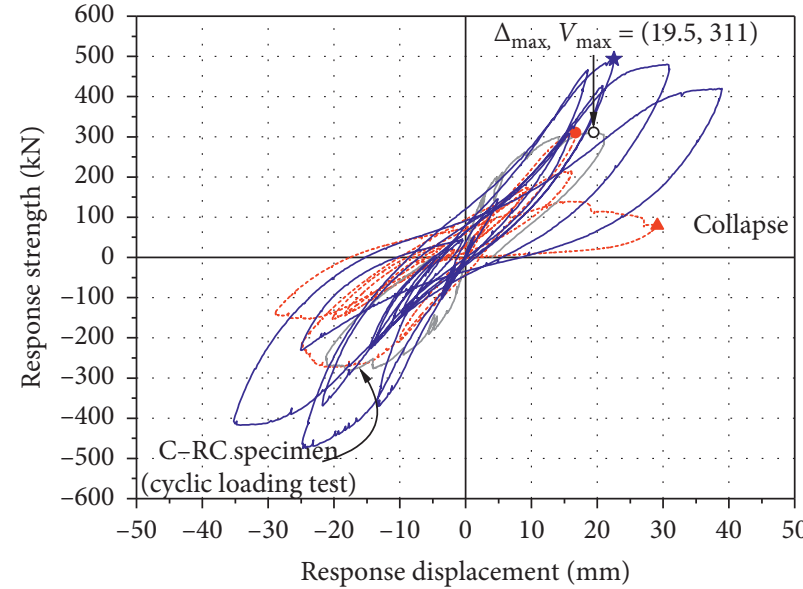

- Maximum response $(16.7 \mathrm{~mm}, 310 \mathrm{kN})$ PD-RC specimen (psedodynamic test, $300 \mathrm{gal})$

$\star \quad$ Maximum response (23.0 mm, $493 \mathrm{kN})$

- PD-BERCF specimen (psedodynamic test, 450 gal)

(b)

FIGURE 14: Lateral response load-displacement relationships for specimens strengthened with the ESRCF system during pseudodynamic testing at 450 gal. (a) PD-WERCF; (b) PD-BERCF.

specimens, earthquake damage was estimated as light. At the highest level of earthquake intensity (450 gal), the welded ESRCF strengthening system showed a maximum response strength of $489 \mathrm{kN}$ with displacement of $26.1 \mathrm{~mm}$, sustaining moderate damage according to the JBDPA [23] and Maeda et al. [11]. As observed in the welded ESRCF specimen, the bolted ESRCF specimen showed moderate damage, with shear cracks observed at a severe earthquake level of $450 \mathrm{gal}$ and a maximum response strength of $493 \mathrm{kN}$ with displacement of $23 \mathrm{~mm}$. Both the welded and bolted ESRCF systems showed fewer and thinner cracks, and no major peeling failure of the concrete cover was observed at $450 \mathrm{gal}$ (unlike the control PD-RC specimen, which collapsed at $300 \mathrm{gal})$.

These results reveal that the external connection methodology using both welded and bolted ESRCF systems 
TABle 6: Comparison among specimens of response strength, response displacement, and degree of earthquake damage following pseudodynamic testing.

\begin{tabular}{|c|c|c|c|c|c|}
\hline Specimen & $\begin{array}{l}\text { Input earthquake } \\
\text { intensity (gal) }\end{array}$ & $\begin{array}{l}\text { Maximum response strength } \\
\text { and strength ratio* } V_{\max }(\mathrm{kN})\end{array}$ & $\begin{array}{l}\text { Response displacement at the } \\
\text { maximum point } \Delta_{\max }(\mathrm{mm})\end{array}$ & $\begin{array}{l}\text { Failure } \\
\text { mode }\end{array}$ & $\begin{array}{l}\text { Degree of } \\
\text { earthquake } \\
\text { damage** }^{*}\end{array}$ \\
\hline \multirow{2}{*}{ PD-RC } & 250 & 305 & 11.9 & $\begin{array}{l}\text { Shear } \\
\text { crack }\end{array}$ & Heavy damage \\
\hline & 300 & 310 & 16.7 & $\begin{array}{l}\text { Shear } \\
\text { failure }\end{array}$ & Collapse \\
\hline \multirow{3}{*}{$\begin{array}{l}\text { PD- } \\
\text { WERCF }\end{array}$} & 300 & $362(1.17=362 / 310)^{*}$ & 9.0 & \multirow{3}{*}{$\begin{array}{l}\text { Shear } \\
\text { crack }\end{array}$} & Slight damage \\
\hline & 375 & $472(1.52=472 / 310)^{*}$ & 21.1 & & Light damage \\
\hline & 450 & $489(1.58=489 / 310)^{*}$ & 26.1 & & Moderate damage \\
\hline \multirow{3}{*}{$\begin{array}{l}\text { PD- } \\
\text { BERCF }\end{array}$} & 300 & $381(1.22=381 / 310)^{*}$ & 9.1 & \multirow{3}{*}{$\begin{array}{l}\text { Shear } \\
\text { crack }\end{array}$} & Slight damage \\
\hline & 375 & $435(1.40=435 / 310)^{*}$ & 23.6 & & Light damage \\
\hline & 450 & $493(1.59=493 / 310)^{*}$ & 23.0 & & Moderate damage \\
\hline
\end{tabular}

${ }^{*}$ Strength ratio is the maximum response strength of a specimen strengthened with ESRCF at each earthquake intensity level relative to that of the control specimen at 300 gal. ${ }^{* *}$ The degree of earthquake damage was estimated based on results reported by JBDPA [23] and Maeda et al. [11].

is useful for seismic strengthening of building systems, especially in terms of increasing ultimate strength.

4.2. Response Displacement-Time History Relationships and Maximum Response Displacement. At 250 gal, the maximum response displacement of the PD-RC control specimen occurred at $4.8 \mathrm{~s}$, with a lateral drift of $14.7 \mathrm{~mm}$; heavy damage was observed. At 300 gal, the control PD-RC specimen showed a maximum response after $5.4 \mathrm{~s}$ with lateral displacement of $29.1 \mathrm{~mm}$, resulting in shear collapse of both columns. Figure 15 shows the response displacement-time history relationships of the PD-RC control during pseudodynamic testing under the 250 and 300 gal NS ground motion recorded at El Centro during the 1940 Imperial Valley earthquake.

The strengthened PD-WERCF specimen, on the other hand, suffered only slight damage, with a maximum response displacement of $8.9 \mathrm{~mm}$ at $2.6 \mathrm{~s}$. The response displacement-time history of the PD-BERCF specimen strengthened using bolted ESRCF was very similar to that of the PD-WERCF specimen under the same earthquake intensity level. At $375 \mathrm{gal}$, the PD-WERCF specimen showed a maximum response displacement at $4.5 \mathrm{~s}$, with a lateral drift of $23.5 \mathrm{~mm}$, resulting in light damage; this result was similar to that of the PD-BERCF specimen, which showed a lateral drift of $20.9 \mathrm{~mm}$ after $4.6 \mathrm{~s}$. At the highest level of earthquake intensity ( $450 \mathrm{gal})$, the PDWERCF specimen strengthened with the welded ESRCF system showed a maximum response displacement of $43.0 \mathrm{~mm}$ at $5.3 \mathrm{~s}$ and suffered moderate damage. Similar to the response displacement-time history of the welded ESRCF specimen, the bolted ESRCF specimen showed moderate damage, with shear cracks occurring at 450 gal and a maximum response displacement of $38.6 \mathrm{~mm}$ after $5.2 \mathrm{~s}$. Figures 16-18 compare response displacement-time history relationships between the PD-WERCF and PDBERCF specimens during pseudodynamic testing at 300 , 375 , and 450 gal, respectively, as well as that of the PD-RC control specimen at $300 \mathrm{gal}$.
The earthquake response parameter of primary interest is the decrease in maximum response displacement of the specimens strengthened by the ESRCF system relative to the control. Table 7 lists these values as percentages of ground acceleration at $300 \mathrm{gal}$, allowing comparison of maximum response displacement between the PD-RC control and PD-ERCF-reinforced specimens. Specimens reinforced using welded and bolted ESRCF systems (PDWERCF and PS-BERCF) had displacement responses approximately $70 \%$ lower than those of the nonstrengthened control specimen.

4.3. Crack and Failure Patterns. Crack and failure patterns that appeared in the PD-RC, PD-WESRCF, and PDBESRCF specimens during pseudodynamic testing were investigated, as well as in the C-RC specimen during the cyclic loading test. Following the pseudodynamic and cyclic loading tests, both specimens failed in response to shear strain. However, structural behavior, including the failure pattern in the final stage for the control (PD-RC and C-RC) and reinforced (PD-WESRCF and PD-BESRCF) specimens, differed significantly. The welded and bolted ESRCF specimens exhibited similar evidence of failure, i.e., similar crack appearances, load-drift relations, and earthquake responses. In the following discussion, we focus on crack and failure patterns in terms of lateral displacement.

4.3.1. Cyclic Loading Test of the Nonstrengthened Control Specimen $(C-R C)$. The control specimen was designed based on the frame of a typical Korean RC building [17] lacking seismic data, under the expectation that it would exhibit shear failure. The cyclic loading test was conducted on the $\mathrm{C}-\mathrm{RC}$ specimen to investigate the hysteresis characteristics of the Korean RC frame lacking seismic data and to determine the variables for the pseudodynamic test. Figure 19 shows the crack patterns observed on the C-RC specimen following the final stages of the cyclic loading test.

The first crack occurred at a positive load of $39 \mathrm{kN}$, and a small flexural crack appeared in both the bottom and upper 


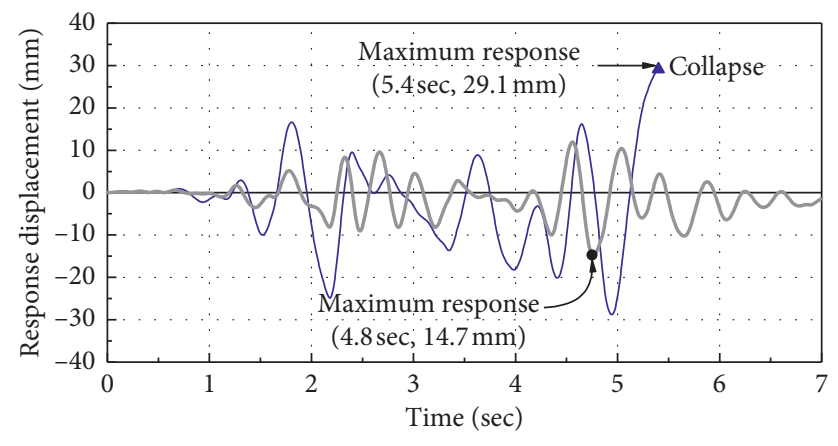

- PD-RC-250gal

- PD-RC-300gal

FIgURE 15: Response displacement-time history relationships of the PD-RC control specimen during pseudodynamic testing at 250 and 300 gal.

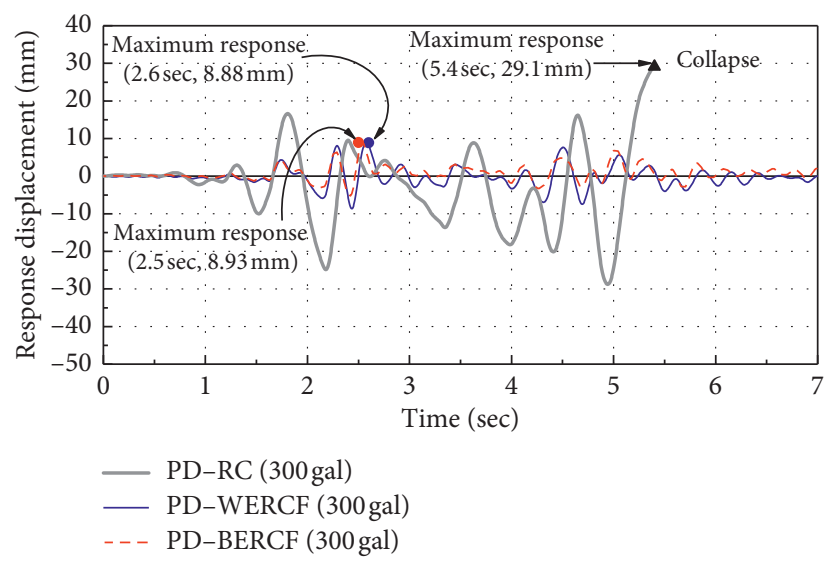

FIGURE 16: Response displacement-time history relationships of the control specimen and ESRCF-strengthened specimens during pseudodynamic testing at 300 gal.

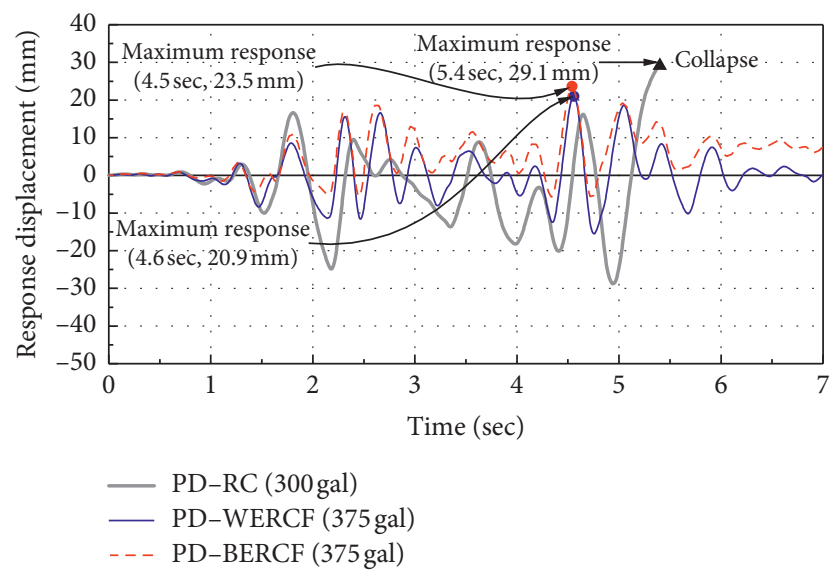

FIGURE 17: Response displacement-time history relationships of the control specimen and ESRCF-strengthened specimens during pseudodynamic testing at 375 gal.

column faces after three cycles during the third loading step $(R=0.2 \%, 2.8 \mathrm{~mm})$. Cracking was not observed in the center of the column. Flexural cracks extended into the middle of the column after step four. Following the seventh loading step $(R=0.5 \%, 7 \mathrm{~mm})$, at both positive $(234 \mathrm{kN})$ and negative $(-211 \mathrm{kN})$ loads, shear cracks were observed at the top faces of the columns, and a number of diagonal shear cracks appeared, some of which were more than $3 \mathrm{~mm}$ wide.

When the applied load reached $271 \mathrm{kN}$, at the ninth positive loading step $(R=1.0 \%, 14 \mathrm{~mm})$, additional shear 


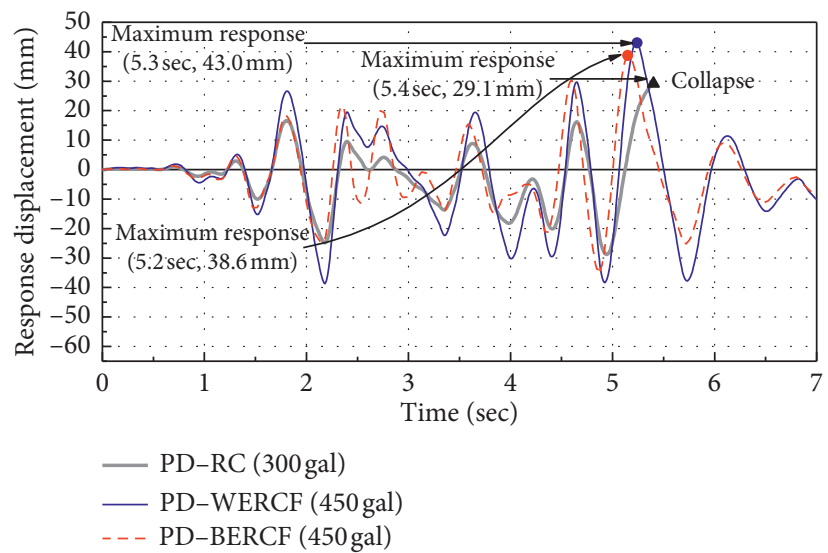

FIGURE 18: Response displacement-time history relationships of the control specimen and ESRCF-strengthened specimens during pseudodynamic testing at 450 gal.

Table 7: Maximum response displacement at 300 gal and the decrease thereof for test specimens in comparison with the control.

\begin{tabular}{lcc}
\hline Specimen & $\begin{array}{c}\text { Maximum response displacement } \\
(\mathrm{mm})\end{array}$ & $\begin{array}{c}\text { Decrease in response displacement of ESRCF-strengthened specimens relative to } \\
\text { control }(\%)\end{array}$ \\
\hline PD-RC & 29.1 & - \\
PD- & 8.88 & 69.5 \\
WERCF & 8.93 & 69.3 \\
PD-BERCF & & - \\
\hline
\end{tabular}

cracks were observed, with increasingly large widths. We observed peeling failure due to shear forces emanating from the concrete cover, likely due to insufficient shear confinement. Shear collapse occurred at the top of both columns following the application of a load of $302 \mathrm{kN}$, with a lateral drift of $21 \mathrm{~mm}(R=1.5 \%)$. The maximum load capacity of the frame of the C-RC specimen was a positive load of $311 \mathrm{kN}$, with a lateral drift of $19.5 \mathrm{~mm}(R=1.4 \%)$. The maximum positive load capacity $(281 \mathrm{kN})$ was similar to the maximum negative load capacity, with a lateral drift of $18.2 \mathrm{~mm}$.

\subsubsection{Pseudodynamic Test of the Nonstrengthened Control} Specimen (PD-RC). As previously stated, ground motion acceleration rates of 250 and 300 gal approximately correspond to the seismic load in Zone-1 under $S_{\mathrm{D}}$ and $S_{\mathrm{E}}$ soil conditions, under which two-thirds of the earthquake ground motion have a $2 \%$ probability of exceedance within 50 years, as specified in the KBC [4]. The first crack was observed under a positive load of $154 \mathrm{kN}$, with a lateral drift of $5.0 \mathrm{~mm}$ within approximately $1.8 \mathrm{~s}$ for an earthquake ground motion of 250 gal. The crack was a small flexural crack, which appeared in the bottom and upper column faces. Flexural cracks extended into the middle of the column after $2.2 \mathrm{~s}$. After $2.5 \mathrm{~s}$, under a negative load of $170 \mathrm{kN}$ with lateral displacement of $8.0 \mathrm{~mm}$, diagonal shear cracks were observed at the top and bottom faces of the columns. Shear cracks increased in number and extended to the middle of both columns after $2.5 \mathrm{~s}$. The maximum earthquake response of the PD-RC control specimen tested using an earthquake ground motion of 250 gal was observed at $4.8 \mathrm{~s}$ under a positive load of $305 \mathrm{kN}$ and lateral displacement of $11.9 \mathrm{~mm}$. The pseudodynamic test was performed for $7 \mathrm{~s}$. Figure 20 shows the crack pattern observed in the PD-RC specimen during the pseudodynamic test, performed by applying earthquake ground motions of $250 \mathrm{gal}$ (El Centro, NS).

In the 300-gal earthquake ground motion test, after approximately $1.52 \mathrm{~s}$, under a negative load of $204 \mathrm{kN}$ with lateral displacement of $10.0 \mathrm{~mm}$, large, visible shear cracks were observed on all faces of the columns. Shear cracks increased in number and extended in width after $1.8 \mathrm{~s}$, with lateral displacement of $16.6 \mathrm{~mm}$. After $2.18 \mathrm{~s}$, with lateral displacement of $24.9 \mathrm{~mm}$, we observed peeling failure due to earthquake shear forces from the concrete cover at the top faces of the columns. Shear collapse occurred at the tops of both columns within $5.4 \mathrm{~s}$, following the application of a positive load of $80 \mathrm{kN}$, with lateral displacement of $28.9 \mathrm{~mm}$. Figure 21 shows the crack pattern observed in the PD-RC specimen during the pseudodynamic test, performed by applying earthquake ground motions of $300 \mathrm{gal}$ (El Centro, NS).

4.3.3. Pseudodynamic Testing of Specimen Strengthened with the Welded ESRCF System (PD-WESRCF). The PD-WESRCF specimen featured an RC frame strengthened by the welded ESRCF system (Figure 2). Figures 22-24 show the sequence of cracks observed in the PD-WESRCF specimen during pseudodynamic testing, using normalized acceleration values of 300,375 , and 450 gal, respectively, based on NS ground motion recorded at El Centro. As stated above, ground motion of 375 and 450 gal was additionally applied to determine the seismic strengthening effectiveness of the 


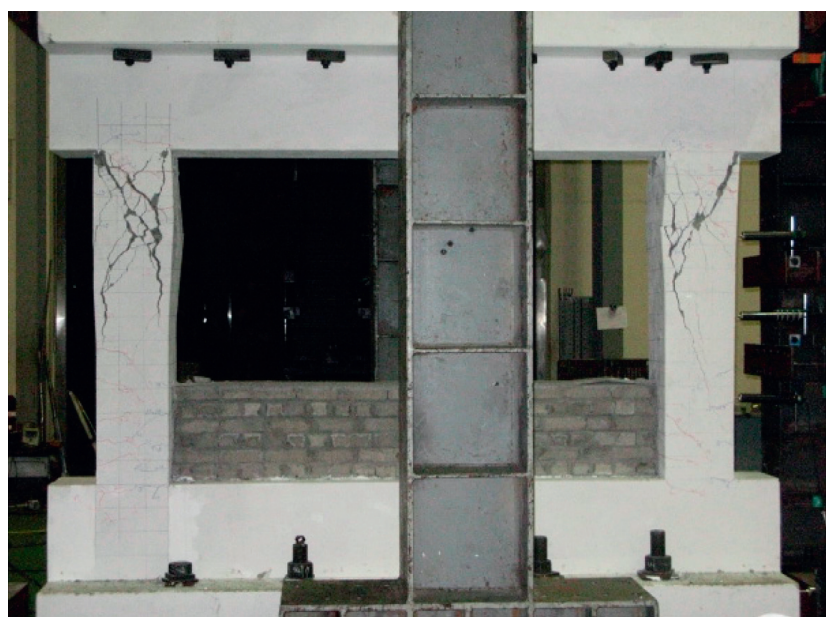

Figure 19: Cracks observed in the C-RC specimen during cyclic loading.

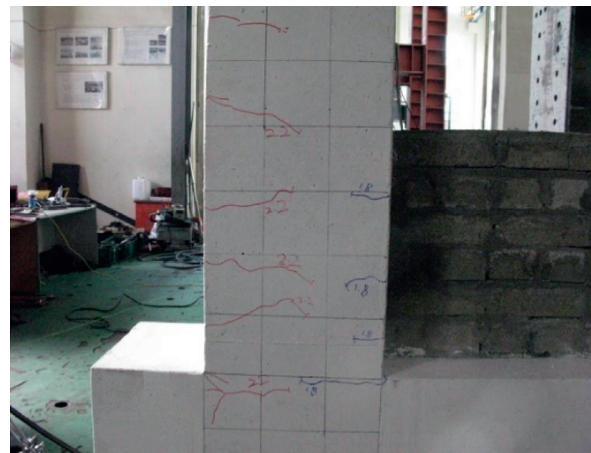

(a)

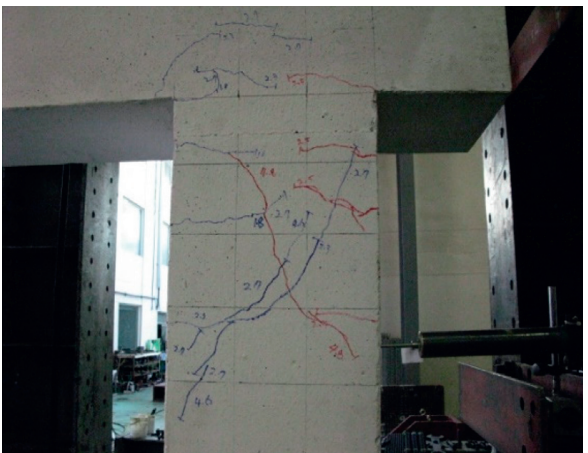

(b)

FIGURE 20: Cracks appeared in the PD-F specimen during pseudodynamic testing under earthquake ground motion of 250 gal, after (a) $2.2 \mathrm{~s}$ with lateral displacement of $7.2 \mathrm{~mm}$ and force of $-166 \mathrm{kN}$ and (b) $4.8 \mathrm{~s}$ with lateral displacement of $13.6 \mathrm{~mm}$ and force of $-212 \mathrm{kN}$.

ESRCF system in the context of a very strong earthquake, under which all earthquake ground motion has a $2 \%$ probability of exceedance within 50 years.

For the 300-gal earthquake ground motion, a small flexural crack was observed in the upper faces of the columns within approximately $1.6 \mathrm{~s}$, under a positive load of $250 \mathrm{kN}$ with lateral displacement of $4.0 \mathrm{~mm}$. Flexural cracks extended to the middle of the column after $2.28 \mathrm{~s}$, with lateral positive displacement of $8.1 \mathrm{~mm}$. However, the flexural crack remained small, and no shear cracks were observed; only one small flexural crack was noted under maximum ground motion, occurring at $2.57 \mathrm{~s}$ under a positive load of $404 \mathrm{kN}$ with lateral displacement of $8.8 \mathrm{~mm}$. After approximately $4.5 \mathrm{~s}$, under a positive load of $320 \mathrm{kN}$ with lateral displacement of $7.32 \mathrm{~mm}$, diagonal shear cracks were observed at the top faces of the columns; however, they were very small, as shown in Figure 22(a). The PD-WESRCF specimen sustained light damage (slight shear and flexural cracks) at 300 gal, corresponding to the seismic load in Zone-1 under $S_{\mathrm{E}}$ soil conditions, as specified in the KBC [4]. The PD-RC control specimen collapsed following the development of large shear cracks. The welded ESRCF strengthening system proposed in this study showed good reinforcement effectiveness when subjected to the maximum earthquake ground motion considered in the KBC.

With earthquake ground motion of 375 gal, after $1.77 \mathrm{~s}$, under a positive load of $368 \mathrm{kN}$ with lateral displacement of $8.6 \mathrm{~mm}$, diagonal shear cracks were observed at the top and bottom faces of the columns. Shear cracks increased in number and extended to the middle of both columns after $4.5 \mathrm{~s}$; however, the cracks remained small, with widths $<1 \mathrm{~mm}$. In contrast, under severe earthquake ground motion (450 gal), under which all earthquake ground motion has with a $2 \%$ probability of exceedance within 50 years, visible shear cracks were observed on all faces of the columns, increasing in number and width $(>2 \mathrm{~mm})$ after $2.16 \mathrm{~s}$ with lateral displacement of $33 \mathrm{~mm}(641 \mathrm{kN})$. Compared with the control PD-RC specimen, which collapsed at 300 gal, the cracks were fewer and thinner, and major peeling failure of the concrete cover was not observed. Thus, the reinforced PD-WESRCF specimen showed moderate damage (i.e., shear cracks) under a severe earthquake level of 450 gal; greater strength was observed in comparison with a nonstrengthened control PD-RC specimen, in which major earthquake damage and shear failure were observed in both upper columns. 


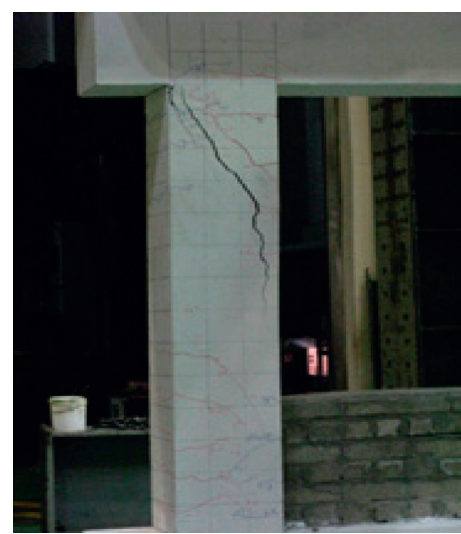

(a)

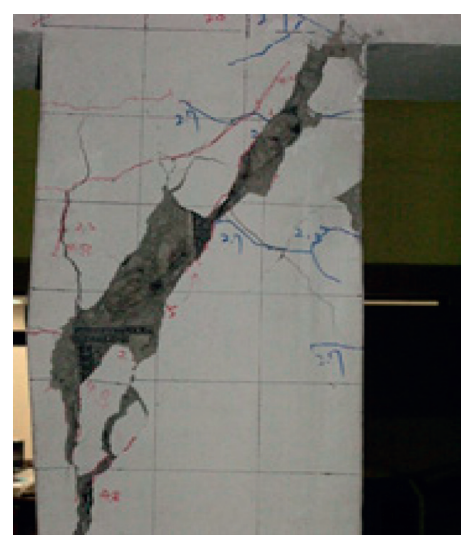

(b)

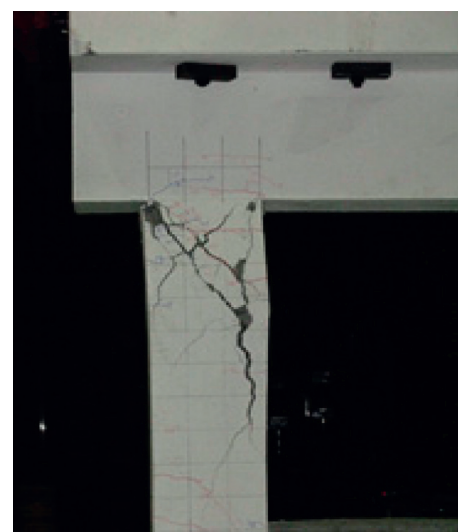

(c)

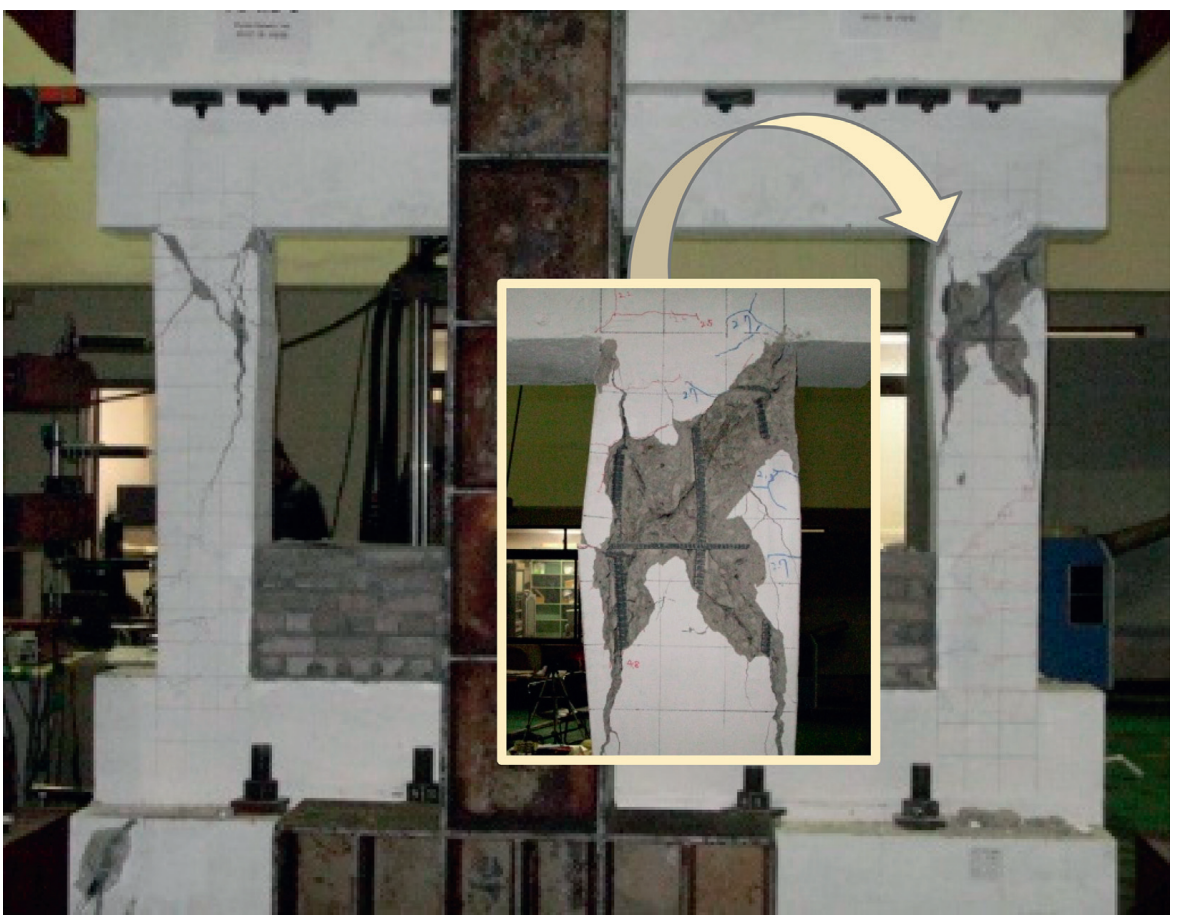

(d)

Figure 21: Cracks appeared in the PD-F specimen during pseudodynamic testing at $300 \mathrm{gal}$, after (a) $1.52 \mathrm{~s}$ with lateral displacement of $10 \mathrm{~mm}$ and force of $-204 \mathrm{kN}$, (b) $2.18 \mathrm{~s}$ with lateral displacement of $24.9 \mathrm{~mm}$ and force of $-268 \mathrm{kN}$, (c) $5.4 \mathrm{~s}$ with lateral displacement of $28.9 \mathrm{~mm}$ and force of $80 \mathrm{kN}$, and (d) during the final stage $(7 \mathrm{~s})$.

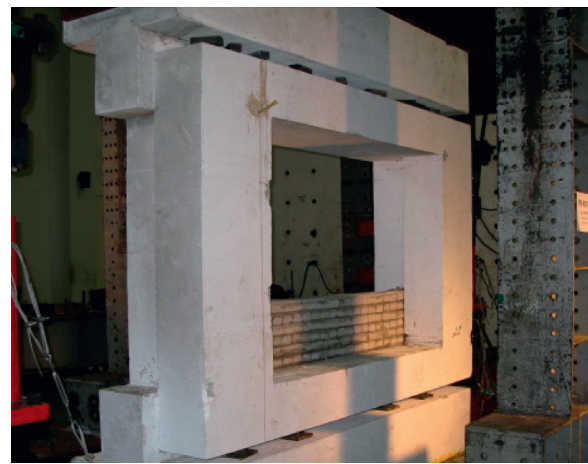

(a)

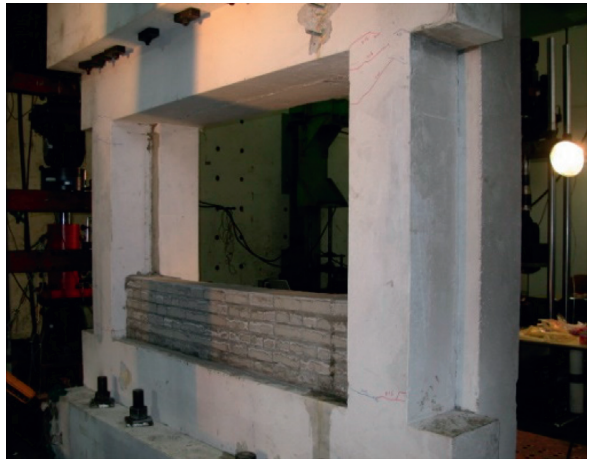

(b)

Figure 22: Cracks appeared in the PD-WESRCF specimen during pseudodynamic testing at 300 gal after (a) $4.5 \mathrm{~s}$ with lateral displacement of $7.32 \mathrm{~mm}$ and force of $320 \mathrm{kN}$, and (b) during the final stage (7 s). 


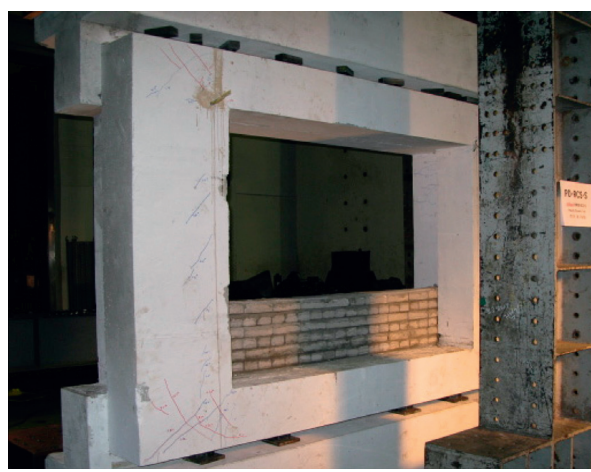

(a)

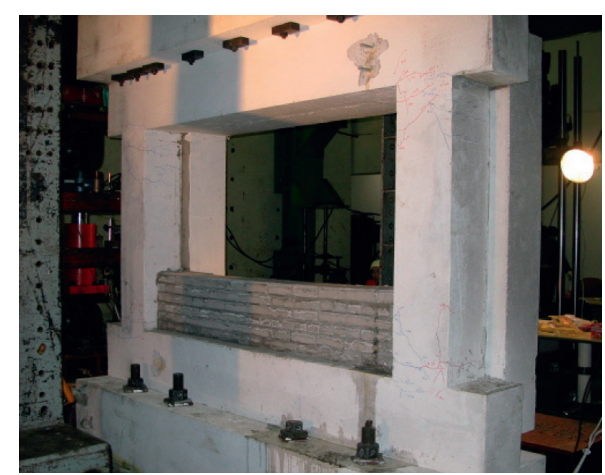

(b)

FIGURE 23: Cracks appeared in the PD-WESRCF specimen during pseudodynamic testing at 400 gal after (a) $4.5 \mathrm{~s}$ with lateral displacement of $19.6 \mathrm{~mm}$ and force of $489 \mathrm{kN}$, and (b) during the final stage (7 s).

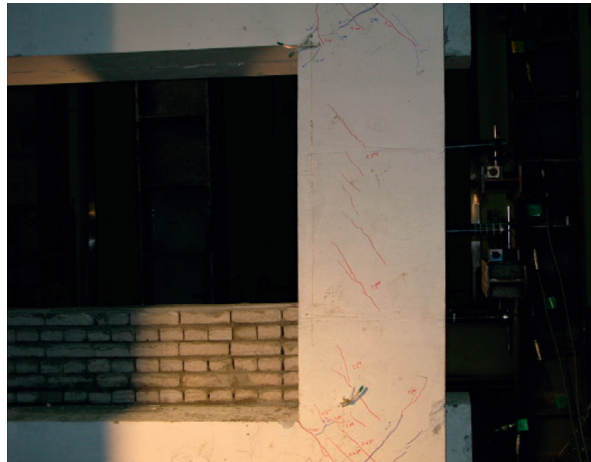

(a)

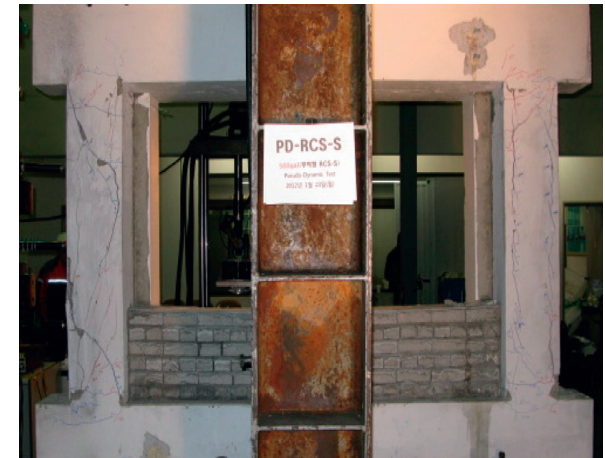

(b)

FIGURE 24: Cracks appeared in the PD-WESRCF specimen during pseudodynamic testing at 500 gal after (a) $2.16 \mathrm{~s}$ with lateral displacement of $33 \mathrm{~mm}$ and force of $-641 \mathrm{kN}$, and (b) during the final stage (7 s).

4.3.4. Pseudodynamic Testing of the Specimen Strengthened with the Bolted ESRCF System (PD-BESRCF). The PDBESRCF specimen was strengthened by the bolted ESRCF system (Figure 3). Generally, crack appearance, load-drift relationships, and earthquake responses of the bolted ESRCF specimen were very similar to those of the welded ESRCF specimen at all earthquake intensity levels (300, 375, and 450 gal). Figures 25-27 show the sequences of cracks observed in the PD-BESRCF specimen during pseudodynamic testing using normalized acceleration values of 300, 375, and 450 gal, respectively, based on the NS ground motion recorded at $\mathrm{El}$ Centro.

At 300 gal, a small flexural crack was observed in the upper faces of the columns with lateral displacement of $3.9 \mathrm{~mm}$ at approximately $1.76 \mathrm{~s}$. The flexural cracks extended to the middle of each column after $2.28 \mathrm{~s}$, with lateral positive displacement of $6.6 \mathrm{~mm}$. However, the flexural cracks remained small, and no shear cracks were observed. After approximately $2.55 \mathrm{~s}$, under a positive load of $381 \mathrm{kN}$ with lateral displacement of $8.9 \mathrm{~mm}$, very slight diagonal shear cracks were observed at the top faces of the columns (Figure 25(a)).
With earthquake ground motion of 375 gal, after $2.17 \mathrm{~s}$, under a negative load of $372 \mathrm{kN}$ with lateral displacement of $5.2 \mathrm{~mm}$, diagonal shear cracks were observed at the top and bottom faces of the columns. Shear cracks increased in number, extended to the middle of both columns after $2.65 \mathrm{~s}$, and remained small ( $<1 \mathrm{~mm}$ wide). The bolted ESRCF strengthening system proposed in this study showed good reinforcement effectiveness when subjected to the maximum earthquake ground motion considered in the KBC. Under severe earthquake ground motion (450 gal, Figure 27), visible shear cracks were observed on all faces of the columns, increasing in number and width $(>2 \mathrm{~mm})$ after $2.2 \mathrm{~s}$ with lateral displacement of $26 \mathrm{~mm}$. Compared with the control PD-RC specimen, which collapsed at 300 gal, the cracks were fewer and thinner, and no major peeling failure of the concrete cover was observed.

As observed for the welded ESRCF specimen, the reinforced PD-WESRCF specimen showed moderate damage, with shear cracks observed under severe earthquake ground motion (450 gal). Greater strength was noted compared with the nonstrengthened control PD-RC specimen, which showed major earthquake damage with shear failure in both upper columns. 


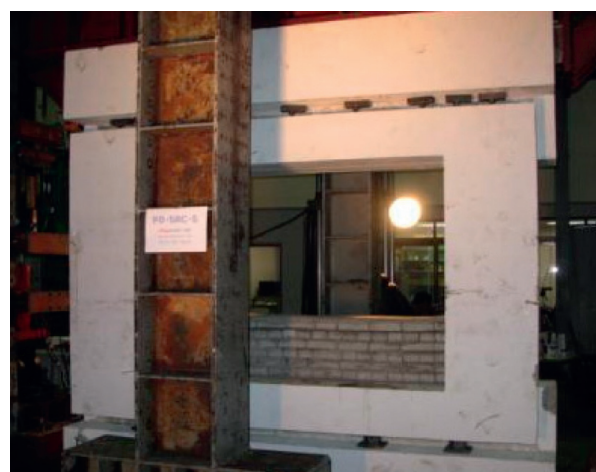

(a)

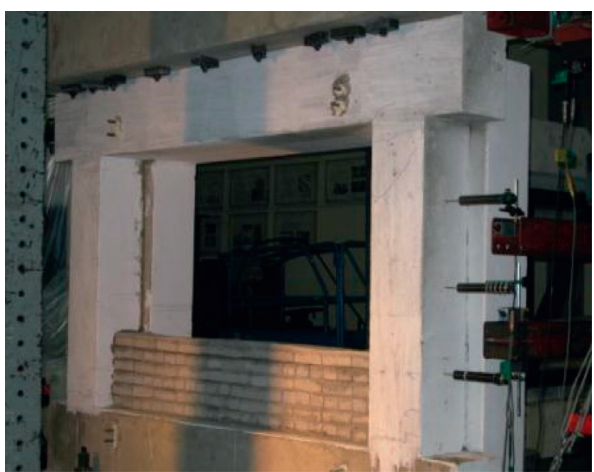

(b)

FIGURE 25: Cracks appeared in the PD-BESRCF specimen during pseudodynamic testing at 300 gal after (a) $2.55 \mathrm{~s}$ with lateral displacement of $8.9 \mathrm{~mm}$ and force of $381 \mathrm{kN}$, and (b) during the final stage $(7 \mathrm{~s})$.

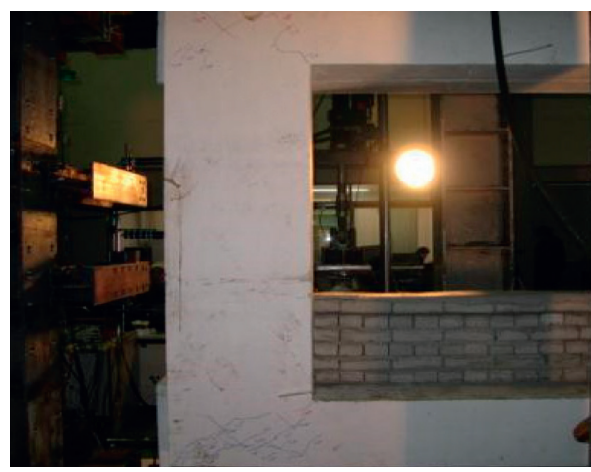

(a)

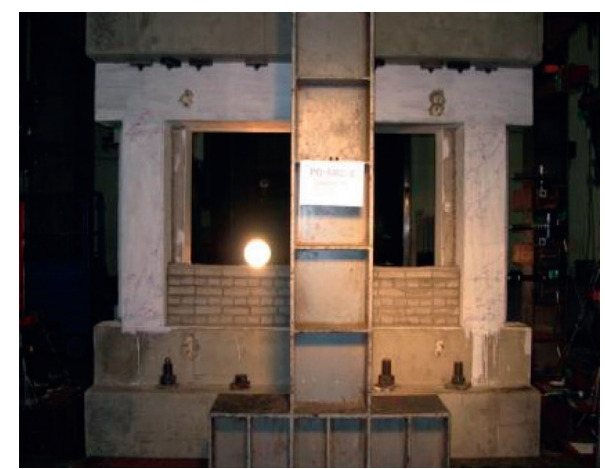

(b)

FIGURE 26: Cracks appeared in the PD-BESRCF specimen during pseudodynamic testing at 375 gal after (a) $2.65 \mathrm{~s}$ with lateral displacement of $19.1 \mathrm{~mm}$ and force of $401 \mathrm{kN}$, and (b) during the final stage (7 s).

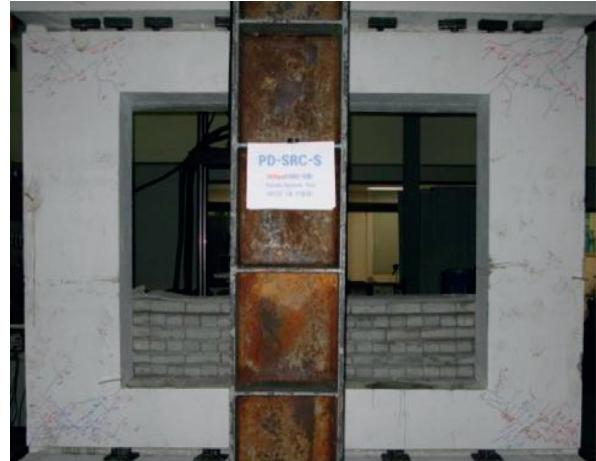

(a)

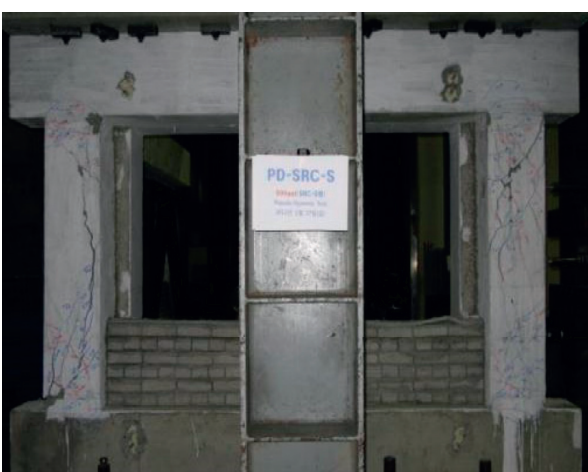

(b)

FIGURE 27: Cracks appeared in the PD-BESRCF specimen during pseudodynamic testing at 450 gal after (a) $1.5 \mathrm{~s}$ with lateral displacement of $13.5 \mathrm{~mm}$ and force of $-372 \mathrm{kN}$, and (b) during the final stage (7 s).

\section{Conclusion}

In this study, we introduced a new seismic retrofitting approach involving the external attachment of steel RC frames. We applied two methods (bolting and welding) to connect existing RC frames to external strengthening elements, with the goal of overcoming the drawbacks associated with conventional seismic retrofitting technology and thus increasing the ultimate strength of existing medium-to-lowrise $\mathrm{RC}$ frame systems lacking seismic data. A typical RC 
frame building constructed prior to the seismic code revision in Korea was selected to assess the seismic strengthening effectiveness of the ESRCF system proposed in this study. We tested specimens designed and fabricated based on the RC frame and strengthened with the ESRCF system. Pseudodynamic and cyclic loading tests were performed to verify the seismic strengthening effectiveness of the proposed ESRCF method, compared with an original control frame, in terms of lateral load-displacement capacity, maximum response strength, response displacement, and degree of earthquake damage under earthquake ground motions specified by the KBC [4]. The test results revealed that when externally installed on the RC frame, the proposed ESRCF retrofitting system effectively increased lateral ultimate strength, leading to marked resistance under a large-scale earthquake. The major results of this work are summarized as follows

(1) The ultimate load capacity of the nonstrengthened control C-RC specimen during the cyclic loading test was $311 \mathrm{kN}$ under a positive load with a lateral drift of $19.5 \mathrm{~mm}(R=1.4 \%)$. Shear collapse finally occurred at the tops of both columns following the application of a load of $302 \mathrm{kN}$, with a lateral drift of $21 \mathrm{~mm}(R=1.5 \%)$. The failure mode at ultimate state was shear, which represents useful information for identifying the failure mechanisms of existing Korean RC buildings without seismic details.

(2) The maximum earthquake response of the PD-RC control specimen at $250 \mathrm{gal}$ was observed at a load of $305 \mathrm{kN}$ with lateral displacement of $11.9 \mathrm{~mm}$, which corresponds to heavy earthquake damage. However, at $300 \mathrm{gal}$, the PD-RC specimen showed a maximum response strength of $310 \mathrm{kN}$ with lateral displacement of $16.7 \mathrm{~mm}$, similar to the C-RC specimen during the cyclic loading test. The top of both columns of the control specimen collapsed after $5.4 \mathrm{~s}$, following the application of a positive load of $80 \mathrm{kN}$, with lateral displacement of $28.9 \mathrm{~mm}$.

(3) For the PD-WERCF specimen, reinforced using the welded ESRCF system, the pseudodynamic test at $300 \mathrm{gal}$ showed a maximum earthquake response at a load of $362 \mathrm{kN}$, with lateral displacement of $9.0 \mathrm{~mm}$, corresponding to slight earthquake damage. The earthquake response behavior of, and degree of damage to, the PD-BERCF specimen strengthened using the bolted ESRCF system was similar to that of the PDWERCF specimen at the same earthquake intensity level. However, the nonstrengthened PD-RC specimen sustained severe damage (i.e., collapse) at 300 gal.

(4) Under a severe earthquake ground motion of $375 \mathrm{gal}$, the PD-WERCF specimen showed a maximum earthquake response at a load of $472 \mathrm{kN}$ with lateral displacement of $21.1 \mathrm{~mm}$; this was similar to the PDBERCF specimen, which showed a maximum response at a load of $435 \mathrm{kN}$ and lateral displacement of $23.6 \mathrm{~mm}$. Both specimens exhibited light earthquake damage. At the highest earthquake intensity level (450 gal), the welded ESRCF strengthening system showed a maximum response strength of $489 \mathrm{kN}$ with lateral displacement of $26.1 \mathrm{~mm}$, similar to the bolted ESRCF specimen, which showed a maximum response strength of $493 \mathrm{kN}$ and displacement of $23 \mathrm{~mm}$. Both structures exhibited moderate earthquake damage.

(5) The maximum response displacement of the PD-RC control specimen was $14.7 \mathrm{~mm}$ (heavy damage) at $250 \mathrm{gal}$, and $29.1 \mathrm{~mm}$ at $300 \mathrm{gal}$ (shear collapse). Both of the strengthened specimens (PD-WERCF and PD-BERCF) suffered slight damage, with a maximum response displacement of approximately $9 \mathrm{~mm}$. Under a severe earthquake ground motion of $375 \mathrm{gal}$, the PD-WERCF specimen experienced maximum response displacement at $4.5 \mathrm{~s}$, with a lateral drift of $23.5 \mathrm{~mm}$ and light damage. These results were similar to those of the PD-BERCF specimen, which exhibited a lateral drift of $20.9 \mathrm{~mm}$ after $4.6 \mathrm{~s}$. At the highest earthquake intensity level (450 gal), the PD-WERCF specimen showed a maximum response displacement of $43.0 \mathrm{~mm}$ (moderate damage). The PD-BESRCF specimen also showed moderate damage, with shear cracks occurring at 450 gal, with a maximum response displacement of $38.6 \mathrm{~mm}$.

(6) The decrease in maximum response displacement of the specimens strengthened by the ESRCF system relative to the control during pseudodynamic testing at $300 \mathrm{gal}$ was 69.5 and $69.3 \%$ for the welded and bolted ESRCF systems, respectively.

(7) These test results demonstrate that the bolting and welding methods proposed in this study for ESRCF strengthening effectively increased the lateral ultimate strength of the structures, resulting in a reduction in response displacement under large-scale, intense earthquake conditions. The retrofit method developed in this study can be applied for seismic retrofitting construction, while residents continue to live within the building. The new methods also increase the ultimate lateral load capacity of existing $\mathrm{RC}$ buildings with shear failure mode.

\section{Data Availability}

All datasets generated during this study are available from the corresponding author on reasonable request.

\section{Conflicts of Interest}

The authors declare no conflicts of interest.

\section{Acknowledgments}

This research was supported by the Korea Agency for Infrastructure Technology Advancement grant (20CTAPC15303302) from the Ministry of Land, Infrastructure, and Transport Affairs of the Korean government. 


\section{References}

[1] IBC-International Building Code, 2018 International Building Code, International Code Council, ANSI, Newyork, NY, USA, 2018.

[2] ACI-American Concrete Institute, "Building code requirements for structural concrete and commentary," ACI, MI, USA, ACI 318-414, 2014.

[3] AIJ-Architectural Institute of Japan, Standard for Structural Calculation of Reinforced Concrete Structures, AIJ, Tokyo, Japan, in Japanese, 2010.

[4] KBC-Korean Building Code, Korean Building Code, Architectural Institute of Korea, Seoul, Korea, in Korean, 2016.

[5] FEMA-Federal Emergency Management Agency, Prestandard and Commentary for Seismic Rehabilitation of Buildings, FEMA, Washington, DC, USA, FEMA 356, 2000.

[6] JBDPA-Japan Building Disaster Prevention Association, Standard for Seismic Evaluation of Existing Reinforced Concrete Buildings, Guidelines for Seismic Retrofit of Existing Reinforced Concrete Buildings, and Technical Manual for Seismic Evaluation and Seismic Retrofit of Existing Reinforced Concrete Buildings, JBDPA, Tokyo, Japan, 2005.

[7] SSRG-Seismic Strengthening Research Group, Seismic Strengthening of RC Buildings, SSRG, Ohmsha Press, Tokyo, Japan, in Japanese, 2008.

[8] K. S. Lee, "An experimental study on hybrid noncompression CF bracing and GF sheet wrapping reinforcement method to restore damaged RC structures," Shock and Vibration, vol. 2015, Article ID 202751, 13 pages, 2015.

[9] J. S. Hwang and K. S. Lee, "Seismic strengthening effects based on pseudodynamic testing of a reinforced concrete building retrofitted with a wire-woven bulk kagome truss damper," Shock and Vibration, vol. 2016, Article ID 3956126, 17 pages, 2016.

[10] AIJ-Architectural Institute of Japan, "Report on damage due to the 1968 Tokachi-Oki earthquake," AIJ, Tokyo, Japan, in Japanese, 1968.

[11] M. Maeda, Y. Nakano, and K. S. Lee, "Postearthquake damage evaluation for RC buildings based on residual seismic capacity," in Proceedings of 13th World Conference on Earthquake Engineering, vol. 1179, Vancouver, Canada, August 2004.

[12] K. S. Lee, Y. Nakano, H. Kumazawa, and T. Okada, "Seismic capacity of reinforced concrete buildings damaged by the 1995 Hyogo-ken Nambu earthquake," Bulletin of Earthquake Resistant Structure Research Center, Vol. 28, Institute of Industrial Science, University of Tokyo, Tokyo, Japan, 1995.

[13] K. S. Lee, "Seismic capacity requirements for low-rise reinforced concrete buildings controlled by both shear and flexure," Journal of Advanced Concrete Technology, vol. 8, no. 1, pp. 75-91, 2010.

[14] FEMA-Federal Emergency Management Agency, Handbook for Seismic Evaluation of Buildings-A Prestandard, FEMA, Washington, DC, USA, FEMA 310, 1998.

[15] K. S. Lee, J. D. Wi, Y. I. Kim, and H. H. Lee, "Seismic safety evaluation of Korean R/C school buildings built in the 1980s," Journal of the Korean Institute for Structural Maintenance Inspection, vol. 13, no. 5, pp. 1-11, 2009, in Korean.

[16] H. Umemura, Earthquake-resistant Design of Reinforced Concrete Buildings, Accounting for the Dynamic Effects of Earthquakes, Giho-do Publishing Co., Tokyo, Japan, in Japanese, 1973.

[17] K. S. Lee and S. W. Shin, "A new methodology for performancebased seismic evaluation of low-rise reinforced concrete buildings using nonlinear required strengths," Journal of Advanced Concrete Technology, vol. 11, no. 4, pp. 151-166, 2013.

[18] K. Takanashi, K. Udagawa, and H. Tanaka, "Pseudo-dynamic tests on a 2-storey steel frame by a computer-load test apparatus hybrid system," in Proceedings of the 7th World Conference on Earthquake Engineering, vol. 7, pp. 225-232, Istanbul, Turkey, September 1980.

[19] Tokyo Soki Kenkyujo Company, Tokyo, Japan, 2020, http:// www.tml.jp/e/.

[20] MTS, Pseudodynamic Testing for 793 Controllers, MTS Systems Corporation, Eden Prairie, MI, USA, 2002.

[21] P. B. Shing, M. Nakashima, and O. S. Bursi, "Application of pseudodynamic test method to structural research," Earthquake Spectra, vol. 12, no. 1, pp. 29-56, 1996.

[22] H. M. Hilber, T. J. R. Hughes, and R. L. Taylor, "Improved numerical dissipation for time integration algorithms in structural dynamics," Earthquake Engineering \& Structural Dynamics, vol. 5, no. 3, pp. 283-292, 1977.

[23] JBDPA-Japan Building Disaster Prevention Association, Standard for Damage Level Classification, JBDPA, Tokyo, Japan, 2001. 